\title{
Fuzzy Fixed Point Results For $\Phi$ Contractive Mapping with Applications
}

\author{
Humaira, ${ }^{1}$ Muhammad Sarwar $\left(D,{ }^{1}\right.$ and G. N. V. Kishore ${ }^{(D)}{ }^{2}$ \\ ${ }^{1}$ Department of Mathematics, University of Malakand, Chakdara, Khyber Pakhtunkhwa, Pakistan \\ ${ }^{2}$ Department of Mathematics, KL University, Vaddeswaram, Guntur, Andhra Pradesh 522 502, India \\ Correspondence should be addressed to Muhammad Sarwar; sarwarswati@gmail.com \\ and G. N. V. Kishore; kishore.apr2@gmail.com
}

Received 12 June 2017; Revised 24 August 2017; Accepted 14 September 2017; Published 9 January 2018

Academic Editor: Rosana Rodríguez López

Copyright (c) 2018 Humaira et al. This is an open access article distributed under the Creative Commons Attribution License, which permits unrestricted use, distribution, and reproduction in any medium, provided the original work is properly cited.

In this paper, using rational type contractions, common fuzzy fixed point result for $\Phi$ contractive mappings involving control functions as coefficients of contractions in the setting of complex-valued metric space is established. The derived results generalizes some result in the existing literature. To show the validity of the derived results an appropriate example and applications are also discussed.

\section{Introduction}

Fixed point theory is considered to be the most interesting and dynamic area of research in the development of nonlinear analysis. In this area, Banach contraction principal [1] is an initiative for researchers during last few decades. This principal plays an important and key role in investigating the existence and uniqueness of solution to various problems in mathematics, physics, engineering, medicines, and social sciences which leads to mathematical models design by system of nonlinear integral equations, functional equations, and differential equations. Banach contraction principal has been generalized in different directions by changing the condition of contraction or by the underlying space. For instance, we refer to [2-8]. Particularly Dass and Gupta [9] extended the Banach contraction principal for rational type inequality and obtained fixed point results in metric space, which is further extended to different spaces by many authors. In the meanwhile researchers realized that where division occurs in cone metric spaces, the concept of rational type contraction is not meaningful.

To overcome this problem a new metric space was recently established by Azam et al. [10], known as complex-valued metric space, where the author obtained fixed point results via rational type contractive condition. This work was further extended by Sitthikul and Saejung [11]. Afterwards Rouzkard and Imdad [12] extended the aforementioned results of Azam et al. by obtaining common fixed point results which satisfies certain rational contractions in complex-valued metrics spaces. Consequently in $[13,14]$, the authors extended common fixed point results for multivalued mappings in complex-valued metric space. In addition, Sintunavarat and Kumam [15] derived common fixed point results by substituting the constant coefficients in contractive condition by control functions.

Heilpern [16] established the concept of fuzzy mappings and obtained fixed point results in metric linear space. He generalized the results of $[1,17]$, under the consideration of fuzzy mappings in complete metric linear spaces. Several mathematicians extended the work of Heilpern in different metric spaces for linear contraction. For instance, we refer to [18-24]. While in [25], the author investigated for fuzzy common fixed point with rational contractive condition. The concept of fuzziness is helpful in solving such real world problems where uncertainty occurs and many authors solve such problems by mathematical modeling in terms of fuzzy differential equations. For instance in [26], the author investigated the existence of solution for fuzzy differential equations. Nieto [27] worked on Cauchy problems for continuous fuzzy differential equations. Song et al. studied the global existence of solutions to fuzzy differential equation [28]. Moreover, the existence of fuzzy solution of first order initial value problem 
was studied in [29], which is lately extended to integrodifferential equations [30]. Recently Long et al. [31] combined the matrix convergent to zero technique with calculations of fuzzy-valued functions, which is quite a new approach to study the system of differential and partial differential equations (PDE's) in generalized fuzzy metric spaces. In [32] Long et al. improved different results existing in the literature on the existence of coincidence points for a pair of mappings and studied applications to partial differential equations with uncertainty. After the wide study of fuzziness in the system of differential equations, it has now been studied in fractional differential equations to obtain the existence and uniqueness of fuzzy solution under Caputo generalized Hukuhara differentiability; for instance, see [33].

In the current work, using rational type contraction, common fuzzy fixed point results for $\Phi$ contractive mappings are studied. The established results generalizes some results from the exiting literature particularly the result of Joshi et al. [34] for fuzzy mappings. Applications and appropriate example are also provided.

\section{Preliminaries}

Definition 1 (see [10]). Assume $\mathfrak{C}$ is the set of complex numbers. For $\varepsilon_{1}, \varepsilon_{2} \in \mathfrak{C}$ we define a partial order $\lesssim$ on $\mathfrak{E}$ as follows:

$$
\begin{aligned}
& \text { (Ci) } \varepsilon_{1} \precsim \varepsilon_{2} \Leftrightarrow \operatorname{Re}\left(\varepsilon_{1}\right) \leq \operatorname{Re}\left(\varepsilon_{2}\right) \text { and } \operatorname{Im}\left(\varepsilon_{1}\right) \leq \operatorname{Im}\left(\varepsilon_{2}\right) ; \\
& \text { (Cii) } \varepsilon_{1} \prec \varepsilon_{2} \Leftrightarrow \operatorname{Re}\left(\varepsilon_{1}\right)<\operatorname{Re}\left(\varepsilon_{2}\right) \text { and } \operatorname{Im}\left(\varepsilon_{1}\right)<\operatorname{Im}\left(\varepsilon_{2}\right) ; \\
& \text { (Ciii) } \varepsilon_{1} \precsim \varepsilon_{2} \Leftrightarrow \operatorname{Re}\left(\varepsilon_{1}\right)=\operatorname{Re}\left(\varepsilon_{2}\right) \text { and } \operatorname{Im}\left(\varepsilon_{1}\right)<\operatorname{Im}\left(\varepsilon_{2}\right) ; \\
& \text { (Civ) } \varepsilon_{1}=\varepsilon_{2} \Leftrightarrow \operatorname{Re}\left(\varepsilon_{1}\right)=\operatorname{Re}\left(\varepsilon_{2}\right) \text { and } \operatorname{Im}\left(\varepsilon_{1}\right)=\operatorname{Im}\left(\varepsilon_{2}\right) \text {. }
\end{aligned}
$$

Clearly if $a \leq b, \Rightarrow a z \precsim b z$, for all $z \in \mathfrak{V}$ and for all $a, b \in \mathscr{R}$. Note that if $\varepsilon_{1} \neq \varepsilon_{2}$ and one of (Ci), (Cii) and (Ciii) is satisfied then $\varepsilon_{1} \preccurlyeq \varepsilon_{2}$, and we write $\varepsilon_{1}=\varepsilon_{2}$ if only (Civ) is satisfied. Note that

$$
\begin{aligned}
& \text { (i) } 0 \precsim \varepsilon_{1} \precsim \varepsilon_{2} \Rightarrow\left|\varepsilon_{1}\right|<\left|\varepsilon_{2}\right|, \forall \varepsilon_{1}, \varepsilon_{2} \in \mathfrak{S} ; \\
& \text { (ii) } \varepsilon_{1} \precsim \varepsilon_{2} \text { and } \varepsilon_{2} \prec \varepsilon_{3} \Rightarrow \varepsilon_{1} \prec \varepsilon_{3}, \forall \varepsilon_{1}, \varepsilon_{2}, \varepsilon_{3} \in \mathfrak{S} \text {. }
\end{aligned}
$$

Definition 2 (see [10]). Let $\mathscr{X}$ be a nonempty set and $d$ : $x \times X \rightarrow \mathbb{C}$ be a mapping which satisfies the following conditions:

(1) $0 \precsim \rho(z, w)$, for all $z, w \in \mathscr{X}$ and $\rho(z, w)=0$ if and only if $z=w$;

(2) $\rho(z, w)=\rho(w, z)$, for all $z, w \in \mathscr{X}$;

(3) $\rho(z, w) \lesssim \rho\left(z, z_{1}\right)+\rho\left(z_{1}, w\right)$, for all $z, z_{1}, w \in X$.

Then $(\mathscr{X}, \rho)$ is called a complex-valued metric space.

Definition 3 (see [10]). A point $z \in \mathscr{X}$ is known as an interior point of a set $Z \subseteq \mathscr{X}$, if we find $0 \prec \epsilon \in \mathfrak{C}$ such that

$$
\mathfrak{B}(z, \epsilon)=\{w \in \mathscr{X}: \rho(z, w) \prec \epsilon\} \subseteq Z .
$$

A point $z \in Z$ is known as the limit point of $Z$, if there exists an open ball $\mathfrak{B}(z, \epsilon)$ such that

$$
\mathfrak{B}(z, \epsilon) \cap(Z \backslash\{z\}) \neq \phi,
$$

where $0 \prec \epsilon \in \mathbb{C}$. A subset $Z$ of $\mathscr{X}$ is said to be open if each point of $Z$ is an interior point of $Z$. Furthermore, $Z$ is said to be closed if it contain all its limit points.

The family

$$
\mathscr{B}=\{\mathfrak{B}(z, \epsilon): z \in \mathscr{X}, 0 \prec \epsilon\}
$$

is a subbasis for a Hausdorff topology $\mathfrak{T}$ on $\mathscr{X}$.

Now recall some definitions from $[13,14]$.

Let $(\mathscr{X}, \rho)$ be a complex-valued metric space. Throughout this paper we denoted the family of all nonempty closed bounded subsets of complex-valued metric space $\mathscr{X}$ by $\mathscr{C} \mathscr{B}(\mathscr{X})$. For $v \in \mathfrak{C}$ we represent

$$
s(\nu)=\{z \in \mathbb{E}: \nu \preceq z\}
$$

and for $w \in \mathscr{X}$ and $B \in \mathscr{C} \mathscr{B}(\mathscr{X})$.

$$
s(w, B)=\bigcup_{b \in B} s(\rho(w, b))=\bigcup_{b \in B}\{z \in \mathfrak{S}: \rho(w, b) \preceq z\} .
$$

For $A, B \in \mathscr{C} \mathscr{B}(\mathscr{X})$, we denote

$$
s(A, B)=\left(\bigcap_{p \in A} s(p, B)\right) \cap\left(\bigcap_{q \in B} s(q, A)\right) .
$$

Let $\tau$ be a multivalued mapping from $\mathscr{X}$ into $\mathscr{C} \mathscr{B}(\mathscr{X})$; for $z \epsilon$ $\mathscr{X}$ and $Q \in \mathscr{C} \mathscr{B}(\mathscr{X})$ we define

$$
\mathscr{W}_{z}(Q)=\{\rho(z, q): q \in Q\} .
$$

Thus for $z, w \in \mathscr{X}$

$$
\mathscr{W}_{z}(\tau w)=\{\rho(z, u): u \in \tau w\}
$$

Lemma 4 (see [35]). Let $(\mathscr{X}, \rho)$ be complex-valued metric space.

(i) Let $z, w \in \mathfrak{C}$. If $z \preceq w$, then $s(z) \subset s(w)$.

(ii) Let $z \in \mathscr{X}$ and $D \in \mathbb{N}(\mathscr{X})$. If $\delta \in s(z, D)$, then $z \in D$.

(iii) Let $w \in \mathfrak{C}, P, Q \in \mathscr{C} \mathscr{B}(\mathscr{X})$ and $p \in P$. If $v \in s(P, Q)$, then $z \in s(p, Q)$ for all $p \in P$ or $z \in s(P, q)$ for all $q \in Q$.

Definition 5 (see [10]). Let $\left\{w_{r}\right\}$ be a sequence in complexvalued metric space $(\mathscr{X}, \rho)$ and $w \in \mathscr{X}$; then

(i) $w$ is a limit point of $\left\{w_{r}\right\}$ if for each $0 \prec \epsilon \in \mathbb{C}$ there exists $r_{0} \in N$ such that $\rho\left(w_{r}, w\right) \preceq \epsilon$ for all $r \geq r_{0}$ and it is written as $\lim _{r \rightarrow \infty} w_{r}=w$.

(ii) $\left\{w_{r}\right\}$ is a Cauchy sequence if for any $0 \prec \epsilon \in \mathbb{C}$ there exists $r_{0} \in N$ such that $\rho\left(w_{r}, w_{r+t}\right) \prec \epsilon$ for all $r>r_{0}$ where $t \in N$.

(iii) we say that $(\mathscr{X}, \rho)$ is complete complex-valued metric space if every Cauchy sequence in $\mathscr{X}$ converges to a point in $\mathscr{X}$ 
Definition 6 (see [18]). Let $(V, \rho)$ be a metric space. A fuzzy set $B$ is characterized by its membership function $f_{B}$ : $V \rightarrow[0,1]$. A set of elements of $V$ along with its grade of membership is called a fuzzy set. For simplicity we denote $f_{B}(u)$ by $B(u)$. The $\alpha$-level set of a fuzzy set $B$ is mentioned by $[B]_{\alpha}$ and is defined as follows:

$$
\begin{aligned}
& {[B]_{\alpha}=\{u: B(u) \geq \alpha\} \quad \text { if } \alpha \in(0,1],} \\
& {[B]_{0}=\{\overline{u: B(u)>0}\} .}
\end{aligned}
$$

Definition 7 (see [18]). Let $\mathfrak{L}(\mathscr{X})$ be the family of all fuzzy sets in a metric space $\mathscr{X}$. For $G, H \in \mathfrak{L}(\mathscr{X}), G \subset H$ means $G(z) \leq$ $H(z)$ for each $z \in \mathscr{X}$.

Definition 8 (see [16]). Assume $\mathscr{X}$ is an arbitrary set and $Y$ is a metric space. A mapping $G$ is called a fuzzy mapping if $G: \mathscr{X} \rightarrow \mathfrak{Q}(Y)$. A fuzzy mapping $G$ is a fuzzy subset on $\mathscr{X} \times Y$ with a membership function $G(x)(y)$. The function $G(x)(y)$ is the grade of membership of $y$ in $G(x)$.

Definition 9 (see [20]). Assume that $(\mathscr{X}, \rho)$ is complex-valued metric space and $G_{1}, G_{2}: \mathscr{X} \rightarrow \mathfrak{L}(\mathscr{X})$ are fuzzy mappings. A point $w \in \mathscr{X}$ is a fuzzy fixed point of $G_{1}$ if $w \in\left[G_{1} w\right]_{\alpha}$ where $\alpha \in[0,1]$ and a common fuzzy fixed point of $G_{1}, G_{2}$ if $w \in\left[G_{1} w\right]_{\alpha} \cap\left[G_{2} w\right]_{\alpha}$. If $\alpha=1$ then $w$ is known as common fixed point of fuzzy mappings.

Definition 10 (see [14]). Suppose $(\mathscr{X}, \rho)$ is complex-valued metric space; the fuzzy mapping $G_{1}: \mathscr{X} \rightarrow \mathfrak{Q}(\mathscr{X})$ enjoys the greatest lower bound property (glb property) on $(\mathscr{X}, \rho)$, if, for any $w \in \mathscr{X}$ and $\alpha \in(0,1]$, the greatest lower bound of $W_{w}\left(\left[G_{1} y\right]_{\alpha}\right)$ exists in $\mathscr{C}$ for all $w, y \in \mathscr{X}$. Here we mention $\rho\left(w,\left[G_{1} y\right]_{\alpha}\right)$ by the glb of $W_{w}\left(\left[G_{1} y\right]_{\alpha}\right)$. That is,

$$
\rho\left(w,\left[G_{1} y\right]_{\alpha}\right)=\inf \left\{\rho(w, u): u \in\left[G_{1} y\right]_{\alpha}\right\} .
$$

Remark 11 (see [13]). Let $(\mathscr{X}, \rho)$ be a complex-valued metric space. If $\mathfrak{S}=\mathbb{R}$, then $(\mathscr{X}, \rho)$ is a metric space. Furthermore $H(A, B)=\inf s(A, B)$ is the Hausdorff distance induced by $\rho$, where $A, B \in \mathscr{C} \mathscr{B}(\mathscr{X})$.

Definition 12 (see [34]). Suppose $\Psi$ is a collection of nondecreasing functions, $\Phi: \mathfrak{E} \rightarrow \mathfrak{C}$, such that $\Phi(0)=0$ and $\Phi(t) \prec t$, when $0 \prec t$.

\section{Main Result}

In this section we present our main results. To present the main results we need the lemmas given below.

Lemma 13. Let $(\mathscr{X}, \rho)$ be complex-valued metric space and $G_{1}, G_{2}: \mathscr{X} \rightarrow \mathfrak{L}(\mathscr{X})$ be fuzzy mappings, such that for each $w \in \mathscr{X}$ and some $\alpha \in(0,1]$ there exists $\left[G_{1} w\right]_{\alpha},\left[G_{2} w\right]_{\alpha}$, nonempty closed and bounded subsets of $\mathscr{X}$. Let $w_{0} \in \mathscr{X}$ and define the sequence $\left\{w_{k}\right\}$ by

$$
\begin{aligned}
& w_{2 k+1} \in\left[G_{1} w_{2 k}\right]_{\alpha}, \\
& w_{2 k+2} \in\left[G_{2} w_{2 k+1}\right]_{\alpha},
\end{aligned}
$$

$$
\forall k=0,1,2, \ldots
$$

Assume that there exists a mapping $\phi: X \rightarrow[0,1)$ such that $\phi(u) \preceq \phi(w)$ for all $u \in\left[G_{1} w\right]_{\alpha}$ and $\phi(v) \preceq \phi(w)$ for all $v \in$ $\left[G_{2} w\right]_{\alpha}$. Then $\phi\left(w_{2 k}\right) \preceq \phi\left(w_{0}\right)$ and $\phi\left(w_{2 k+1}\right) \preceq \phi\left(w_{1}\right)$.

Proof. Suppose $w \in \mathscr{X}$ and $k=0,1,2, \ldots$. Then we have

$$
\begin{aligned}
\phi\left(w_{2 k}\right) & \leq \phi\left(w_{2 k-2}\right) \quad \text { for } w_{2 k-1} \in\left[G_{1} w_{2 k-2}\right]_{\alpha}, \\
& \leq \phi\left(w_{2 k-4}\right) \quad \text { for } w_{2 k-2} \in\left[G_{1} w_{2 k-4}\right]_{\alpha}, \\
& \leq \cdots \preceq \phi\left(w_{0}\right) .
\end{aligned}
$$

Similarly we have

$$
\phi\left(w_{2 k+1}\right) \preceq \phi\left(w_{1}\right) .
$$

Theorem 14. Suppose $(X, \rho)$ is a complete complex-valued metric space and $G_{1}, G_{2}: \mathscr{X} \rightarrow \mathfrak{L}(\mathscr{X})$ are fuzzy mappings satisfying glb property. Assume that for each $y \in \mathscr{X}$ and some $\alpha \in(0,1]$ there exist $\left[G_{1} y\right]_{\alpha},\left[G_{2} y\right]_{\alpha}$ which are nonempty closed bounded subsets of $\mathscr{X}$. Suppose there exist mappings $\psi_{i}: \mathscr{X} \rightarrow[0,1), i=1, \ldots, 7$ such that

(i) $\psi_{i}(u) \preceq \psi_{i}(y), i=1, \ldots, 7$ for all $u \in\left[G_{1} y\right]_{\alpha}$ and $y \in \mathscr{X}$

(ii) $\psi_{i}(v) \preceq \psi_{i}(y), i=1, \ldots, 7$ for all $v \in\left[G_{2} y\right]_{\alpha}$ and $y \in X$

(iii) $\sum \psi_{i}(y)+2 \psi_{4}(y)<1, i=1,2,3,6,7 \forall y \in \mathscr{X}$; and

$$
\begin{aligned}
& \Phi\left(\psi_{1}(y) \rho(y, w)+\psi_{2}(y) \rho\left(y,\left[G_{1} y\right]_{\alpha}\right)\right. \\
& +\psi_{3}(y) \rho\left(w,\left[G_{2} w\right]_{\alpha}\right) \\
& +\psi_{4}(y) \frac{\rho\left(y,\left[G_{1} y\right]_{\alpha}\right) \rho\left(y,\left[G_{2} w\right]_{\alpha}\right)}{1+\rho(y, w)} \\
& +\psi_{5}(y) \frac{\rho\left(w,\left[G_{1} y\right]_{\alpha}\right) \rho\left(w,\left[G_{2} w\right]_{\alpha}\right)}{1+\rho(y, w)} \\
& +\psi_{6}(y) \frac{\rho\left(y,\left[G_{1} y\right]_{\alpha}\right) \rho\left(w,\left[G_{2} w\right]_{\alpha}\right)}{1+\rho(y, w)} \\
& \left.\quad+\psi_{7}(y) \frac{\rho\left(w,\left[G_{1} y\right]_{\alpha}\right) \rho\left(y,\left[G_{2} w\right]_{\alpha}\right)}{1+\rho(y, w)}\right) \\
& \quad \in s\left(\left[G_{1} y\right]_{\alpha},\left[G_{2} w\right]_{\alpha}\right)
\end{aligned}
$$

for some $\Phi \in \Psi$ and for all $y, w \in \mathscr{X}$. Then $G_{1}$ and $G_{2}$ have a common fuzzy fixed point. 
Proof. Let $y_{0} \in \mathscr{X}$ and $y_{1} \in\left[G_{1} y_{0}\right]_{\alpha}$. Using (14) with $y=y_{0}$ and $w=y_{1}$ we get

$$
\begin{aligned}
& \Phi\left(\psi_{1}\left(y_{0}\right) \rho\left(y_{0}, y_{1}\right)+\psi_{2}\left(y_{0}\right) \rho\left(y_{0},\left[G_{1} y_{0}\right]_{\alpha}\right)\right. \\
& +\psi_{3}\left(y_{0}\right) \rho\left(y_{1},\left[G_{2} y_{1}\right]_{\alpha}\right) \\
& +\psi_{4}\left(y_{0}\right) \frac{\rho\left(y_{0},\left[G_{1} y_{0}\right]_{\alpha}\right) \rho\left(y_{0},\left[G_{2} y_{1}\right]_{\alpha}\right)}{1+\rho\left(y_{0}, y_{1}\right)} \\
& \quad+\psi_{5}\left(y_{0}\right) \frac{\rho\left(y_{1},\left[G_{1} y_{0}\right]_{\alpha}\right) \rho\left(y_{1},\left[G_{2} y_{1}\right]_{\alpha}\right)}{1+\rho\left(y_{0}, y_{1}\right)} \\
& +\psi_{6}\left(y_{0}\right) \frac{\rho\left(y_{0},\left[G_{1} y_{0}\right]_{\alpha}\right) \rho\left(y_{1},\left[G_{2} y_{1}\right]_{\alpha}\right)}{1+\rho\left(y_{0}, y_{1}\right)} \\
& \left.\quad+\psi_{7}\left(y_{0}\right) \frac{\rho\left(y_{1},\left[G_{1} y_{0}\right]_{\alpha}\right) \rho\left(y_{0},\left[G_{2} y_{1}\right]_{\alpha}\right)}{1+\rho\left(y_{0}, y_{1}\right)}\right) \\
& \quad \in s\left(\left[G_{1} y_{0}\right]_{\alpha},\left[G_{2} y_{1}\right]_{\alpha}\right) .
\end{aligned}
$$

By Lemma 4(iii) we have

$$
\begin{aligned}
& \Phi\left(\psi_{1}\left(y_{0}\right) \rho\left(y_{0}, y_{1}\right)+\psi_{2}\left(y_{0}\right) \rho\left(y_{0},\left[G_{1} y_{0}\right]_{\alpha}\right)\right. \\
& +\psi_{3}\left(y_{0}\right) \rho\left(y_{1},\left[G_{2} y_{1}\right]_{\alpha}\right) \\
& +\psi_{4}\left(y_{0}\right) \frac{\rho\left(y_{0},\left[G_{1} y_{0}\right]_{\alpha}\right) \rho\left(y_{0},\left[G_{2} y_{1}\right]_{\alpha}\right)}{1+\rho\left(y_{0}, y_{1}\right)} \\
& +\psi_{5}\left(y_{0}\right) \frac{\rho\left(y_{1},\left[G_{1} y_{0}\right]_{\alpha}\right) \rho\left(y_{1},\left[G_{2} y_{1}\right]_{\alpha}\right)}{1+\rho\left(y_{0}, y_{1}\right)} \\
& +\psi_{6}\left(y_{0}\right) \frac{\rho\left(y_{0},\left[G_{1} y_{0}\right]_{\alpha}\right) \rho\left(y_{1},\left[G_{2} y_{1}\right]_{\alpha}\right)}{1+\rho\left(y_{0}, y_{1}\right)} \\
& \left.\quad+\psi_{7}\left(y_{0}\right) \frac{\rho\left(y_{1},\left[G_{1} y_{0}\right]_{\alpha}\right) \rho\left(y_{0},\left[G_{2} y_{1}\right]_{\alpha}\right)}{1+\rho\left(y_{0}, y_{1}\right)}\right) \\
& \quad \in s\left(y_{1},\left[G_{2} y_{1}\right]_{\alpha}\right) .
\end{aligned}
$$

By definition there exists some $y_{2} \in\left[G_{2} y_{1}\right]_{\alpha}$, such that

$$
\begin{aligned}
& \Phi\left(\psi_{1}\left(y_{0}\right) \rho\left(y_{0}, y_{1}\right)+\psi_{2}\left(y_{0}\right) \rho\left(y_{0},\left[G_{1} y_{0}\right]_{\alpha}\right)\right. \\
& +\psi_{3}\left(y_{0}\right) \rho\left(y_{1},\left[G_{2} y_{1}\right]_{\alpha}\right) \\
& +\psi_{4}\left(y_{0}\right) \frac{\rho\left(y_{0},\left[G_{1} y_{0}\right]_{\alpha}\right) \rho\left(y_{0},\left[G_{2} y_{1}\right]_{\alpha}\right)}{1+\rho\left(y_{0}, y_{1}\right)} \\
& +\psi_{5}\left(y_{0}\right) \frac{\rho\left(y_{1},\left[G_{1} y_{0}\right]_{\alpha}\right) \rho\left(y_{1},\left[G_{2} y_{1}\right]_{\alpha}\right)}{1+\rho\left(y_{0}, y_{1}\right)}
\end{aligned}
$$

$$
\begin{aligned}
& +\psi_{6}\left(y_{0}\right) \frac{\rho\left(y_{0},\left[G_{1} y_{0}\right]_{\alpha}\right) \rho\left(y_{1},\left[G_{2} y_{1}\right]_{\alpha}\right)}{1+\rho\left(y_{0}, y_{1}\right)} \\
& \left.+\psi_{7}\left(y_{0}\right) \frac{\rho\left(y_{1},\left[G_{1} y_{0}\right]_{\alpha}\right) \rho\left(y_{0},\left[G_{2} y_{1}\right]_{\alpha}\right)}{1+\rho\left(y_{0}, y_{1}\right)}\right) \\
& \in s\left(\rho\left(y_{1}, y_{2}\right)\right) .
\end{aligned}
$$

Therefore

$$
\begin{aligned}
\rho\left(y_{1}, y_{2}\right) \leq \Phi\left(\psi_{1}\left(y_{0}\right) \rho\left(y_{0}, y_{1}\right)\right. \\
+\psi_{2}\left(y_{0}\right) \rho\left(y_{0},\left[G_{1} y_{0}\right]_{\alpha}\right) \\
+\psi_{3}\left(y_{0}\right) \rho\left(y_{1},\left[G_{2} y_{1}\right]_{\alpha}\right) \\
+\psi_{4}\left(y_{0}\right) \frac{\rho\left(y_{0},\left[G_{1} y_{0}\right]_{\alpha}\right) \rho\left(y_{0},\left[G_{2} y_{1}\right]_{\alpha}\right)}{1+\rho\left(y_{0}, y_{1}\right)} \\
+\psi_{5}\left(y_{0}\right) \frac{\rho\left(y_{1},\left[G_{1} y_{0}\right]_{\alpha}\right) \rho\left(y_{1},\left[G_{2} y_{1}\right]_{\alpha}\right)}{1+\rho\left(y_{0}, y_{1}\right)} \\
+\psi_{6}\left(y_{0}\right) \frac{\rho\left(y_{0},\left[G_{1} y_{0}\right]_{\alpha}\right) \rho\left(y_{1},\left[G_{2} y_{1}\right]_{\alpha}\right)}{1+\rho\left(y_{0}, y_{1}\right)} \\
\left.+\psi_{7}\left(y_{0}\right) \frac{\rho\left(y_{1},\left[G_{1} y_{0}\right]_{\alpha}\right) \rho\left(y_{0},\left[G_{2} y_{1}\right]_{\alpha}\right)}{1+\rho\left(y_{0}, y_{1}\right)}\right) \\
\quad \prec \psi_{1}\left(y_{0}\right) \rho\left(y_{0}, y_{1}\right)+\psi_{2}\left(y_{0}\right) \rho\left(y_{0},\left[G_{1} y_{0}\right]_{\alpha}\right) \\
+\psi_{3}\left(y_{0}\right) \rho\left(y_{1},\left[G_{2} y_{1}\right]_{\alpha}\right)+\psi_{4}\left(y_{0}\right) \\
\quad \frac{\rho\left(y_{0},\left[G_{1} y_{0}\right]_{\alpha}\right) \rho\left(y_{0},\left[G_{2} y_{1}\right]_{\alpha}\right)}{1+\rho\left(y_{0}, y_{1}\right)}+\psi_{5}\left(y_{0}\right) \\
+\frac{\rho\left(y_{1},\left[G_{1} y_{0}\right]_{\alpha}\right) \rho\left(y_{0},\left[G_{2} y_{1}\right]_{\alpha}\right)}{1+\rho\left(y_{0}, y_{1}\right)} . \\
+\frac{\rho\left(y_{1},\left[G_{1} y_{0}\right]_{\alpha}\right) \rho\left(y_{1},\left[G_{2} y_{1}\right]_{\alpha}\right)}{1+\rho\left(y_{0}, y_{1}\right)}+\psi_{6}\left(y_{0}\right) \rho\left(y_{1},\left[G_{2} y_{1}\right]_{\alpha}\right) \\
+\psi_{7}\left(y_{0}\right)
\end{aligned}
$$

Using the glb property of $G_{1}$ and $G_{2}$ we have

$$
\begin{aligned}
\rho\left(y_{1}, y_{2}\right) \leq & \psi_{1}\left(y_{0}\right) \rho\left(y_{0}, y_{1}\right)+\psi_{2}\left(y_{0}\right) \rho\left(y_{0}, y_{1}\right) \\
& +\psi_{3}\left(y_{0}\right) \rho\left(y_{1}, y_{2}\right) \\
& +\psi_{4}\left(y_{0}\right) \frac{\rho\left(y_{0}, y_{1}\right) \rho\left(y_{0}, y_{2}\right)}{1+\rho\left(y_{0}, y_{1}\right)} \\
& +\psi_{5}\left(y_{0}\right) \frac{\rho\left(y_{1}, y_{1}\right) \rho\left(y_{1}, y_{2}\right)}{1+\rho\left(y_{0}, y_{1}\right)}
\end{aligned}
$$




$$
\begin{aligned}
& +\psi_{6}\left(y_{0}\right) \frac{\rho\left(y_{0}, y_{1}\right) \rho\left(y_{1}, y_{2}\right)}{1+\rho\left(y_{0}, y_{1}\right)} \\
& +\psi_{7}\left(y_{0}\right) \frac{\rho\left(y_{1}, y_{1}\right) \rho\left(y_{0}, y_{2}\right)}{1+\rho\left(y_{0}, y_{1}\right)} .
\end{aligned}
$$

It implies that

$$
\begin{aligned}
\rho\left(y_{1}, y_{2}\right) \leq & \psi_{1}\left(y_{0}\right) \rho\left(y_{0}, y_{1}\right)+\psi_{2}\left(y_{0}\right) \rho\left(y_{0}, y_{1}\right) \\
& +\psi_{3}\left(y_{0}\right) \rho\left(y_{1}, y_{2}\right)+\psi_{4}\left(y_{0}\right) \rho\left(y_{0}, y_{2}\right) \\
& +\psi_{6}\left(y_{0}\right) \rho\left(y_{1}, y_{2}\right) \\
\leq & \psi_{1}\left(y_{0}\right) \rho\left(y_{0}, y_{1}\right)+\psi_{2}\left(y_{0}\right) \rho\left(y_{0}, y_{1}\right) \\
& +\psi_{3}\left(y_{0}\right) \rho\left(y_{1}, y_{2}\right)+\psi_{4}\left(y_{0}\right) \rho\left(y_{0}, y_{1}\right) \\
& +\psi_{4}\left(y_{0}\right) \rho\left(y_{1}, y_{2}\right)+\psi_{6}\left(y_{0}\right) \rho\left(y_{1}, y_{2}\right) .
\end{aligned}
$$

Finally we get

$$
\begin{gathered}
\rho\left(y_{1}, y_{2}\right) \leq \mu \rho\left(y_{0}, y_{1}\right) \\
\left|\rho\left(y_{1}, y_{2}\right)\right| \leq \mu\left|\rho\left(y_{0}, y_{1}\right)\right|,
\end{gathered}
$$

where

$$
\mu=\frac{\psi_{1}\left(y_{0}\right)+\psi_{2}\left(y_{0}\right)+\psi_{4}\left(y_{0}\right)}{1-\left(\psi_{3}\left(y_{0}\right)+\psi_{4}\left(y_{0}\right)+\psi_{6}\left(y_{0}\right)\right)}<1 .
$$

Now for $y_{2} \in\left[G_{2} y_{1}\right]_{\alpha}$, consider

$$
\begin{aligned}
& \Phi\left(\psi_{1}\left(y_{2}\right) \rho\left(y_{2}, y_{1}\right)+\psi_{2}\left(y_{2}\right) \rho\left(y_{2},\left[G_{1} y_{2}\right]_{\alpha}\right)\right. \\
& +\psi_{3}\left(y_{2}\right) \rho\left(y_{1},\left[G_{2} y_{1}\right]_{\alpha}\right) \\
& +\psi_{4}\left(y_{2}\right) \frac{\rho\left(y_{2},\left[G_{1} y_{2}\right]_{\alpha}\right) \rho\left(y_{2},\left[G_{2} y_{1}\right]_{\alpha}\right)}{1+\rho\left(y_{2}, y_{1}\right)} \\
& +\psi_{5}\left(y_{2}\right) \frac{\rho\left(y_{1},\left[G_{1} y_{2}\right]_{\alpha}\right) \rho\left(y_{1},\left[G_{2} y_{1}\right]_{\alpha}\right)}{1+\rho\left(y_{2}, y_{1}\right)} \\
& +\psi_{6}\left(y_{2}\right) \frac{\rho\left(y_{2},\left[G_{1} y_{2}\right]_{\alpha}\right) \rho\left(y_{1},\left[G_{2} y_{1}\right]_{\alpha}\right)}{1+\rho\left(y_{2}, y_{1}\right)} \\
& \left.\quad+\psi_{7}\left(y_{2}\right) \frac{\rho\left(y_{1},\left[G_{1} y_{2}\right]_{\alpha}\right) \rho\left(y_{2},\left[G_{2} y_{1}\right]_{\alpha}\right)}{1+\rho\left(y_{2}, y_{1}\right)}\right) \\
& \quad \in s\left(\left[G_{1} y_{2}\right]_{\alpha},\left[G_{2} y_{1}\right]_{\alpha}\right) .
\end{aligned}
$$

Using Lemma 4(iii) we get

$$
\begin{aligned}
\Phi & \left(\psi_{1}\left(y_{2}\right) \rho\left(y_{2}, y_{1}\right)+\psi_{2}\left(y_{2}\right) \rho\left(y_{2},\left[G_{1} y_{2}\right]_{\alpha}\right)\right. \\
& +\psi_{3}\left(y_{2}\right) \rho\left(y_{1},\left[G_{2} y_{1}\right]_{\alpha}\right)
\end{aligned}
$$

$$
\begin{aligned}
& +\psi_{4}\left(y_{2}\right) \frac{\rho\left(y_{2},\left[G_{1} y_{2}\right]_{\alpha}\right) \rho\left(y_{2},\left[G_{2} y_{1}\right]_{\alpha}\right)}{1+\rho\left(y_{2}, y_{1}\right)} \\
& +\psi_{5}\left(y_{2}\right) \frac{\rho\left(y_{1},\left[G_{1} y_{2}\right]_{\alpha}\right) \rho\left(y_{1},\left[G_{2} y_{1}\right]_{\alpha}\right)}{1+\rho\left(y_{2}, y_{1}\right)} \\
& +\psi_{6}\left(y_{2}\right) \frac{\rho\left(y_{2},\left[G_{1} y_{2}\right]_{\alpha}\right) \rho\left(y_{1},\left[G_{2} y_{1}\right]_{\alpha}\right)}{1+\rho\left(y_{2}, y_{1}\right)} \\
& \left.+\psi_{7}\left(y_{2}\right) \frac{\rho\left(y_{1},\left[G_{1} y_{2}\right]_{\alpha}\right) \rho\left(y_{2},\left[G_{2} y_{1}\right]_{\alpha}\right)}{1+\rho\left(y_{2}, y_{1}\right)}\right) \\
& \in s\left(\left[G_{1} y_{2}\right]_{\alpha}, y_{2}\right) .
\end{aligned}
$$

By definition there exists $y_{3} \in\left[G_{1} y_{2}\right]_{\alpha}$, such that

$$
\begin{aligned}
& \Phi\left(\psi_{1}\left(y_{2}\right) \rho\left(y_{2}, y_{1}\right)+\psi_{2}\left(y_{2}\right) \rho\left(y_{2},\left[G_{1} y_{2}\right]_{\alpha}\right)\right. \\
& +\psi_{3}\left(y_{2}\right) \rho\left(y_{1},\left[G_{2} y_{1}\right]_{\alpha}\right) \\
& +\psi_{4}\left(y_{2}\right) \frac{\rho\left(y_{2},\left[G_{1} y_{2}\right]_{\alpha}\right) \rho\left(y_{2},\left[G_{2} y_{1}\right]_{\alpha}\right)}{1+\rho\left(y_{2}, y_{1}\right)} \\
& +\psi_{5}\left(y_{2}\right) \frac{\rho\left(y_{1},\left[G_{1} y_{2}\right]_{\alpha}\right) \rho\left(y_{1},\left[G_{2} y_{1}\right]_{\alpha}\right)}{1+\rho\left(y_{2}, y_{1}\right)} \\
& +\psi_{6}\left(y_{2}\right) \frac{\rho\left(y_{2},\left[G_{1} y_{2}\right]_{\alpha}\right) \rho\left(y_{1},\left[G_{2} y_{1}\right]_{\alpha}\right)}{1+\rho\left(y_{2}, y_{1}\right)} \\
& \left.\quad+\psi_{7}\left(y_{2}\right) \frac{\rho\left(y_{1},\left[G_{1} y_{2}\right]_{\alpha}\right) \rho\left(y_{2},\left[G_{2} y_{1}\right]_{\alpha}\right)}{1+\rho\left(y_{2}, y_{1}\right)}\right) \\
& \quad \in s\left(\rho\left(y_{3}, y_{2}\right)\right) .
\end{aligned}
$$

Therefore

$$
\begin{aligned}
& \rho\left(y_{3}, y_{2}\right) \leq \Phi\left(\psi_{1}\left(y_{2}\right) \rho\left(y_{2}, y_{1}\right)\right. \\
& \quad+\psi_{2}\left(y_{2}\right) \rho\left(y_{2},\left[G_{1} y_{2}\right]_{\alpha}\right) \\
& +\psi_{3}\left(y_{2}\right) \rho\left(y_{1},\left[G_{2} y_{1}\right]_{\alpha}\right) \\
& +\psi_{4}\left(y_{2}\right) \frac{\rho\left(y_{2},\left[G_{1} y_{2}\right]_{\alpha}\right) \rho\left(y_{2},\left[G_{2} y_{1}\right]_{\alpha}\right)}{1+\rho\left(y_{2}, y_{1}\right)} \\
& +\psi_{5}\left(y_{2}\right) \frac{\rho\left(y_{1},\left[G_{1} y_{2}\right]_{\alpha}\right) \rho\left(y_{1},\left[G_{2} y_{1}\right]_{\alpha}\right)}{1+\rho\left(y_{2}, y_{1}\right)} \\
& \quad+\psi_{6}\left(y_{2}\right) \frac{\rho\left(y_{2},\left[G_{1} y_{2}\right]_{\alpha}\right) \rho\left(y_{1},\left[G_{2} y_{1}\right]_{\alpha}\right)}{1+\rho\left(y_{2}, y_{1}\right)} \\
& \left.\quad+\psi_{7}\left(y_{2}\right) \frac{\rho\left(y_{1},\left[G_{1} y_{2}\right]_{\alpha}\right) \rho\left(y_{2},\left[G_{2} y_{1}\right]_{\alpha}\right)}{1+\rho\left(y_{2}, y_{1}\right)}\right) \\
& \quad<\psi_{1}\left(y_{2}\right) \rho\left(y_{2}, y_{1}\right)+\psi_{2}\left(y_{2}\right) \rho\left(y_{2},\left[G_{1} y_{2}\right]_{\alpha}\right) \\
& \quad+\psi_{3}\left(y_{2}\right) \rho\left(y_{1},\left[G_{2} y_{1}\right]_{\alpha}\right)+\psi_{4}\left(y_{2}\right)
\end{aligned}
$$




$$
\begin{aligned}
& \cdot \frac{\rho\left(y_{2},\left[G_{1} y_{2}\right]_{\alpha}\right) \rho\left(y_{2},\left[G_{2} y_{1}\right]_{\alpha}\right)}{1+\rho\left(y_{2}, y_{1}\right)}+\psi_{5}\left(y_{2}\right) \\
& \cdot \frac{\rho\left(y_{1},\left[G_{1} y_{2}\right]_{\alpha}\right) \rho\left(y_{1},\left[G_{2} y_{1}\right]_{\alpha}\right)}{1+\rho\left(y_{2}, y_{1}\right)}+\psi_{6}\left(y_{2}\right) \\
& . \frac{\rho\left(y_{2},\left[G_{1} y_{2}\right]_{\alpha}\right) \rho\left(y_{1},\left[G_{2} y_{1}\right]_{\alpha}\right)}{1+\rho\left(y_{2}, y_{1}\right)}+\psi_{7}\left(y_{2}\right) \\
& . \frac{\rho\left(y_{1},\left[G_{1} y_{2}\right]_{\alpha}\right) \rho\left(y_{2},\left[G_{2} y_{1}\right]_{\alpha}\right)}{1+\rho\left(y_{2}, y_{1}\right)} .
\end{aligned}
$$

Again utilizing the greatest lower bound property of $G_{1}$ and $G_{2}$ we get

$$
\begin{aligned}
\rho\left(y_{3}, y_{2}\right) \leq & \psi_{1}\left(y_{2}\right) \rho\left(y_{2}, y_{1}\right)+\psi_{2}\left(y_{2}\right) \rho\left(y_{2}, y_{3}\right) \\
& +\psi_{3}\left(y_{2}\right) \rho\left(y_{1}, y_{2}\right) \\
& +\psi_{4}\left(y_{2}\right) \frac{\rho\left(y_{2}, y_{3}\right) \rho\left(y_{2}, y_{2}\right)}{1+\rho\left(y_{2}, y_{1}\right)} \\
& +\psi_{5}\left(y_{2}\right) \frac{\rho\left(y_{1}, y_{3}\right) \rho\left(y_{1}, y_{2}\right)}{1+\rho\left(y_{2}, y_{1}\right)} \\
& +\psi_{6}\left(y_{2}\right) \frac{\rho\left(y_{2}, y_{3}\right) \rho\left(y_{1}, y_{2}\right)}{1+\rho\left(y_{2}, y_{1}\right)} \\
& +\psi_{7}\left(y_{2}\right) \frac{\rho\left(y_{1}, y_{3}\right) \rho\left(y_{2}, y_{2}\right)}{1+\rho\left(y_{2}, y_{1}\right)}
\end{aligned}
$$

It implies that

$$
\begin{aligned}
\rho\left(y_{3}, y_{2}\right) \leq & \psi_{1}\left(y_{2}\right) \rho\left(y_{2}, y_{1}\right)+\psi_{2}\left(y_{2}\right) \rho\left(y_{2}, y_{3}\right) \\
& +\psi_{3}\left(y_{2}\right) \rho\left(y_{1}, y_{2}\right)+\psi_{5}\left(y_{2}\right) \rho\left(y_{1}, y_{3}\right) \\
& +\psi_{6}\left(y_{2}\right) \rho\left(y_{2}, y_{3}\right) \\
\leq & \psi_{1}\left(y_{2}\right) \rho\left(y_{2}, y_{1}\right)+\psi_{2}\left(y_{2}\right) \rho\left(y_{2}, y_{3}\right) \\
& +\psi_{3}\left(y_{2}\right) \rho\left(y_{1}, y_{2}\right)+\psi_{5}\left(y_{2}\right) \rho\left(y_{1}, y_{2}\right) \\
& +\psi_{5}\left(y_{2}\right) \rho\left(y_{2}, y_{3}\right)+\psi_{6}\left(y_{2}\right) \rho\left(y_{2}, y_{3}\right) .
\end{aligned}
$$

Applying Lemma 13 we get

$$
\begin{aligned}
\rho\left(y_{3}, y_{2}\right) \leq & \psi_{1}\left(y_{0}\right) \rho\left(y_{2}, y_{1}\right)+\psi_{2}\left(y_{0}\right) \rho\left(y_{2}, y_{3}\right) \\
& +\psi_{3}\left(y_{0}\right) \rho\left(y_{1}, y_{2}\right)+\psi_{5}\left(y_{0}\right) \rho\left(y_{1}, y_{2}\right) \\
& +\psi_{5}\left(y_{0}\right) \rho\left(y_{2}, y_{3}\right)+\psi_{6}\left(y_{0}\right) \rho\left(y_{2}, y_{3}\right) .
\end{aligned}
$$

Finally we get

$$
\begin{gathered}
\rho\left(y_{3}, y_{2}\right) \leq v \rho\left(y_{2}, y_{1}\right) \\
\left|\rho\left(y_{2}, y_{3}\right)\right| \leq \nu\left|\rho\left(y_{1}, y_{2}\right)\right|,
\end{gathered}
$$

where

$$
\nu=\frac{\psi_{1}\left(y_{0}\right)+\psi_{3}\left(y_{0}\right)+\psi_{5}\left(y_{0}\right)}{1-\left(\psi_{2}\left(y_{0}\right)+\psi_{5}\left(y_{0}\right)+\psi_{6}\left(y_{0}\right)\right)}<1 .
$$

Inductively we can obtain a sequence $\left\{y_{n}\right\}$ in $\mathscr{X}$ such that $y_{2 r+1} \in\left[G_{1} x_{2 r}\right]_{\alpha}, y_{2 r+2} \in\left[G_{2} y_{2 r+1}\right]_{\alpha}$ for $r=0,1,2 \ldots$

$$
\begin{aligned}
& \left|\rho\left(y_{2 r+1}, y_{2 r+2}\right)\right| \leq \mu\left|\rho\left(y_{2 r}, y_{2 r+1}\right)\right| \\
& \left|\rho\left(y_{2 r+2}, y_{2 r+3}\right)\right| \leq \nu\left|\rho\left(y_{2 r+1}, y_{2 r+2}\right)\right| .
\end{aligned}
$$

It implies that

$$
\begin{aligned}
\left|\rho\left(y_{2 r+1}, y_{2 r+2}\right)\right| & \leq \mu\left|\rho\left(y_{2 r}, y_{2 r+1}\right)\right| \\
& \leq \mu \nu\left|\rho\left(y_{2 r-1}, y_{2 r}\right)\right| \\
& \leq \mu \nu \mu\left|\rho\left(y_{2 r-2}, y_{2 r-1}\right)\right| \leq \cdots \\
& \leq \mu(\mu \nu)^{r}\left|\rho\left(y_{0}, y_{1}\right)\right| \\
\left|\rho\left(y_{2 r+2}, y_{2 r+3}\right)\right| & \leq \nu\left|\rho\left(y_{2 r+1}, y_{2 r+2}\right)\right| \leq \cdots \\
& \leq(\mu \nu)^{r+1}\left|\rho\left(y_{0}, y_{1}\right)\right| .
\end{aligned}
$$

Then for $s<t$, we have

$$
\begin{aligned}
\rho\left(y_{2 s+1}, y_{2 t+1}\right) \leq & \rho\left(y_{2 s+1}, y_{2 s+2}\right)+\rho\left(y_{2 s+2}, y_{2 s+3}\right) \\
& +\rho\left(y_{2 s+3}, y_{2 s+4}\right)+\cdots \\
& +\rho\left(y_{2 t}, y_{2 t+1}\right)
\end{aligned}
$$

which implies that

$$
\begin{aligned}
& \left|\rho\left(y_{2 s+1}, y_{2 t+1}\right)\right| \\
& \leq\left|\rho\left(y_{2 s+1}, y_{2 s+2}\right)\right|+\left|\rho\left(y_{2 s+2}, y_{2 s+3}\right)\right| \\
& \quad+\left|\rho\left(y_{2 s+3}, y_{2 s+4}\right)\right|+\cdots+\left|\rho\left(y_{2 t}, y_{2 t+1}\right)\right| \\
& \leq\left[\mu \sum_{k=s}^{t-1}(\mu \nu)^{k}+\sum_{k=s+1}^{t}(\mu \nu)^{k}\right]\left|\rho\left(y_{0}, y_{1}\right)\right| .
\end{aligned}
$$

Similarly we obtain

$$
\begin{aligned}
& \rho\left(y_{2 s}, y_{2 t+1}\right) \leq\left[\sum_{k=s}^{t}(\mu \nu)^{k}+\mu \sum_{k=s}^{t-1}(\mu \nu)^{k}\right]\left|\rho\left(y_{0}, y_{1}\right)\right|, \\
& \rho\left(y_{2 s}, y_{2 t}\right) \leq\left[\sum_{k=s}^{t-1}(\mu \nu)^{k}+\mu \sum_{k=s}^{t-1}(\mu \nu)^{k}\right]\left|\rho\left(y_{0}, y_{1}\right)\right| \\
& \rho\left(y_{2 s+1}, y_{2 t}\right) \\
& \leq\left[\mu \sum_{k=s}^{t-1}(\mu \nu)^{k}+\sum_{k=s+1}^{t-1}(\mu \nu)^{k}\right]\left|\rho\left(y_{0}, y_{1}\right)\right| .
\end{aligned}
$$

Since $(\mu \nu)<1$, therefore $\left\{y_{r}\right\}$ is a Cauchy sequence in $\mathscr{X}$. Since $\mathscr{X}$ is complete so there exists $l \in \mathscr{X}$ such that $y_{r} \rightarrow l$ 
when $r \rightarrow \infty$. Now we have to prove that $l \in\left[G_{1} l\right]_{\alpha}$ and $l \in\left[G_{2} l\right]_{\alpha}$. From (14) with $y=y_{2 r}$ and $w=l$ we get

$$
\begin{aligned}
& \Phi\left(\psi_{1}\left(y_{2 r}\right) \rho\left(y_{2 r}, l\right)+\psi_{2}\left(y_{2 r}\right) \rho\left(y_{2 r},\left[G_{1} y_{2 r}\right]_{\alpha}\right)\right. \\
& +\psi_{3}\left(y_{2 r}\right) \rho\left(l,\left[G_{2} l\right]_{\alpha}\right) \\
& +\psi_{4}\left(y_{2 r}\right) \frac{\rho\left(y_{2 r},\left[G_{1} y_{2 r}\right]_{\alpha}\right) \rho\left(y_{2 r},\left[G_{2} l\right]_{\alpha}\right)}{1+\rho\left(y_{2 r}, l\right)} \\
& \quad+\psi_{5}\left(y_{2 r}\right) \frac{\rho\left(l,\left[G_{1} y_{2 r}\right]_{\alpha}\right) \rho\left(l,\left[G_{2} l\right]_{\alpha}\right)}{1+\rho\left(y_{2 r}, l\right)} \\
& +\psi_{6}\left(y_{2 r}\right) \frac{\rho\left(y_{2 r},\left[G_{1} y_{2 r}\right]_{\alpha}\right) \rho\left(l,\left[G_{2} l\right]_{\alpha}\right)}{1+\rho\left(y_{2 r}, l\right)} \\
& \left.\quad+\psi_{7}\left(y_{2 r}\right) \frac{\rho\left(l,\left[G_{1} y_{2 r}\right]_{\alpha}\right) \rho\left(y_{2 r},\left[G_{2} l\right]_{\alpha}\right)}{1+\rho\left(y_{2 r}, l\right)}\right) \\
& \quad \in s\left(\left[G_{1} y_{2 r}\right]_{\alpha},\left[G_{2} l\right]_{\alpha}\right) .
\end{aligned}
$$

Since $y_{2 r+1} \in\left[G_{1} y_{2 r}\right]_{\alpha}$, so by Lemma 4(iii) we have

$$
\begin{aligned}
& \Phi\left(\psi_{1}\left(y_{2 r}\right) \rho\left(y_{2 r}, l\right)+\psi_{2}\left(y_{2 r}\right) \rho\left(y_{2 r},\left[G_{1} y_{2 r}\right]_{\alpha}\right)\right. \\
& +\psi_{3}\left(y_{2 r}\right) \rho\left(l,\left[G_{2} l\right]_{\alpha}\right) \\
& +\psi_{4}\left(y_{2 r}\right) \frac{\rho\left(y_{2 r},\left[G_{1} y_{2 r}\right]_{\alpha}\right) \rho\left(y_{2 r},\left[G_{2} l\right]_{\alpha}\right)}{1+\rho\left(y_{2 r}, l\right)} \\
& \quad+\psi_{5}\left(y_{2 r}\right) \frac{\rho\left(l,\left[G_{1} y_{2 r}\right]_{\alpha}\right) \rho\left(l,\left[G_{2} l\right]_{\alpha}\right)}{1+\rho\left(y_{2 r}, l\right)} \\
& +\psi_{6}\left(y_{2 r}\right) \frac{\rho\left(y_{2 r},\left[G_{1} y_{2 r}\right]_{\alpha}\right) \rho\left(l,\left[G_{2} l\right]_{\alpha}\right)}{1+\rho\left(y_{2 r}, l\right)} \\
& \left.\quad+\psi_{7}\left(y_{2 r}\right) \frac{\rho\left(l,\left[G_{1} y_{2 r}\right]_{\alpha}\right) \rho\left(y_{2 r},\left[G_{2} l\right]_{\alpha}\right)}{1+\rho\left(y_{2 r}, l\right)}\right) \\
& \quad \in s\left(y_{2 r+1},\left[G_{2} l\right]_{\alpha}\right) .
\end{aligned}
$$

By definition there exists some $w_{r} \in\left[G_{2} l\right]_{\alpha}$, such that

$$
\begin{aligned}
& \Phi\left(\psi_{1}\left(y_{2 r}\right) \rho\left(y_{2 r}, l\right)+\psi_{2}\left(y_{2 r}\right) \rho\left(y_{2 r},\left[G_{1} y_{2 r}\right]_{\alpha}\right)\right. \\
& +\psi_{3}\left(y_{2 r}\right) \rho\left(l,\left[G_{2} l\right]_{\alpha}\right) \\
& +\psi_{4}\left(y_{2 r}\right) \frac{\rho\left(y_{2 r},\left[G_{1} y_{2 r}\right]_{\alpha}\right) \rho\left(y_{2 r},\left[G_{2} l\right]_{\alpha}\right)}{1+\rho\left(y_{2 r}, l\right)} \\
& +\psi_{5}\left(y_{2 r}\right) \frac{\rho\left(l,\left[G_{1} y_{2 r}\right]_{\alpha}\right) \rho\left(l,\left[G_{2} l\right]_{\alpha}\right)}{1+\rho\left(y_{2 r}, l\right)} \\
& +\psi_{6}\left(y_{2 r}\right) \frac{\rho\left(y_{2 r},\left[G_{1} y_{2 r}\right]_{\alpha}\right) \rho\left(l,\left[G_{2} l\right]_{\alpha}\right)}{1+\rho\left(y_{2 r}, l\right)}
\end{aligned}
$$

$$
\begin{aligned}
& \left.+\psi_{7}\left(y_{2 r}\right) \frac{\rho\left(l,\left[G_{1} y_{2 r}\right]_{\alpha}\right) \rho\left(y_{2 r},\left[G_{2} l\right]_{\alpha}\right)}{1+\rho\left(y_{2 r}, l\right)}\right) \\
& \in s\left(\rho\left(y_{2 r+1}, w_{r}\right)\right) .
\end{aligned}
$$

Therefore

$$
\begin{aligned}
& \rho\left(y_{2 r+1}, w_{r}\right) \leq \Phi\left(\psi_{1}\left(y_{2 r}\right) \rho\left(y_{2 r}, l\right)\right. \\
& +\psi_{2}\left(y_{2 r}\right) \rho\left(y_{2 r},\left[G_{1} y_{2 r}\right]_{\alpha}\right) \\
& +\psi_{3}\left(y_{2 r}\right) \rho\left(l,\left[G_{2} l\right]_{\alpha}\right) \\
& \quad+\psi_{4}\left(y_{2 r}\right) \frac{\rho\left(y_{2 r},\left[G_{1} y_{2 r}\right]_{\alpha}\right) \rho\left(y_{2 r},\left[G_{2} l\right]_{\alpha}\right)}{1+\rho\left(y_{2 r}, l\right)} \\
& +\psi_{5}\left(y_{2 r}\right) \frac{\rho\left(l,\left[G_{1} y_{2 r}\right]_{\alpha}\right) \rho\left(l,\left[G_{2} l\right]_{\alpha}\right)}{1+\rho\left(y_{2 r}, l\right)} \\
& +\psi_{6}\left(y_{2 r}\right) \frac{\rho\left(y_{2 r},\left[G_{1} y_{2 r}\right]_{\alpha}\right) \rho\left(l,\left[G_{2} l\right]_{\alpha}\right)}{1+\rho\left(y_{2 r}, l\right)} \\
& \left.+\psi_{7}\left(y_{2 r}\right) \frac{\rho\left(l,\left[G_{1} y_{2 r}\right]_{\alpha}\right) \rho\left(y_{2 r},\left[G_{2} l\right]_{\alpha}\right)}{1+\rho\left(y_{2 r}, l\right)}\right) .
\end{aligned}
$$

By using the greatest lower bound property of $G_{1}$ and $G_{2}$, we have

$$
\begin{aligned}
& \rho\left(y_{2 r+1}, w_{r}\right) \leq \Phi\left(\psi_{1}\left(y_{2 r}\right) \rho\left(y_{2 r}, l\right)\right. \\
& +\psi_{2}\left(y_{2 r}\right) \rho\left(y_{2 r}, y_{2 r+1}\right)+\psi_{3}\left(y_{2 r}\right) \rho\left(l, w_{r}\right) \\
& +\psi_{4}\left(y_{2 r}\right) \frac{\rho\left(y_{2 r}, y_{2 r+1}\right) \rho\left(y_{2 r}, w_{r}\right)}{1+\rho\left(y_{2 r}, l\right)} \\
& +\psi_{5}\left(y_{2 r}\right) \frac{\rho\left(l, y_{2 r+1}\right) \rho\left(l, w_{r}\right)}{1+\rho\left(y_{2 r}, l\right)} \\
& +\psi_{6}\left(y_{2 r}\right) \frac{\rho\left(y_{2 r}, y_{2 r+1}\right) \rho\left(l, w_{r}\right)}{1+\rho\left(y_{2 r}, l\right)} \\
& \left.+\psi_{7}\left(y_{2 r}\right) \frac{\rho\left(l, y_{2 r+1}\right) \rho\left(y_{2 r+1}, w_{r}\right)}{1+\rho\left(y_{2 r}, l\right)}\right) \leq \psi_{1}\left(y_{2 r}\right) \\
& +\rho\left(y_{2 r}, l\right)+\psi_{2}\left(y_{2 r}\right) \rho\left(y_{2 r}, y_{2 r+1}\right)+\psi_{3}\left(y_{2 r}\right) \\
& +\rho\left(l, w_{r}\right)+\psi_{4}\left(y_{2 r}\right) \frac{\rho\left(y_{2 r}, y_{2 r+1}\right) \rho\left(y_{2 r}, w_{r}\right)}{1+\rho\left(y_{2 r}, l\right)} \\
& +\psi_{5}\left(y_{2 r}\right) \frac{\rho\left(l, y_{2 r+1}\right) \rho\left(l, w_{r}\right)}{1+\rho\left(y_{2 r}, l\right)}+\psi_{6}\left(y_{2 r}\right) \\
& +\frac{\rho\left(y_{2 r}, y_{2 r+1}\right) \rho\left(l, w_{r}\right)}{1+\rho\left(y_{2 r}, l\right)}+\psi_{7}\left(y_{2 r}\right) \\
& +\frac{\rho\left(l, y_{2 r+1}\right) \rho\left(y_{2 r+1}, w_{r}\right)}{1+\rho\left(y_{2 r}, l\right)} .
\end{aligned}
$$


Now by using triangular inequality, we get

$$
\begin{aligned}
\rho\left(l, w_{r}\right) \preceq & \rho\left(l, y_{2 r+1}\right)+\rho\left(y_{2 r+1}, w_{r}\right) \\
\preceq & \rho\left(l, y_{2 r+1}\right)+\psi_{1}\left(y_{2 r}\right) \rho\left(y_{2 r}, l\right) \\
& +\psi_{2}\left(y_{2 r}\right) \rho\left(y_{2 r}, y_{2 r+1}\right) \\
& +\psi_{3}\left(y_{2 r}\right) \rho\left(l, w_{r}\right) \\
& +\psi_{4}\left(y_{2 r}\right) \frac{\rho\left(y_{2 r}, y_{2 r+1}\right) \rho\left(y_{2 r}, w_{r}\right)}{1+\rho\left(y_{2 r}, l\right)} \\
& +\psi_{5}\left(y_{2 r}\right) \frac{\rho\left(l, y_{2 r+1}\right) \rho\left(l, w_{r}\right)}{1+\rho\left(y_{2 r}, l\right)} \\
& +\psi_{6}\left(y_{2 r}\right) \frac{\rho\left(y_{2 r}, y_{2 r+1}\right) \rho\left(l, w_{r}\right)}{1+\rho\left(y_{2 r}, l\right)} \\
& +\psi_{7}\left(y_{2 r}\right) \frac{\rho\left(l, y_{2 r+1}\right) \rho\left(y_{2 r+1}, w_{r}\right)}{1+\rho\left(y_{2 r}, l\right)} .
\end{aligned}
$$

Applying Lemma 13 we get

$$
\begin{aligned}
\rho\left(l, w_{r}\right) \leq & \rho\left(l, y_{2 r+1}\right)+\rho\left(y_{2 r+1}, w_{r}\right) \\
\leq & \rho\left(l, y_{2 r+1}\right)+\psi_{1}\left(y_{0}\right) \rho\left(y_{2 r}, l\right) \\
& +\psi_{2}\left(y_{0}\right) \rho\left(y_{2 r}, y_{2 r+1}\right)+\psi_{3}\left(y_{0}\right) \rho\left(l, w_{r}\right) \\
& +\psi_{4}\left(y_{0}\right) \frac{\rho\left(y_{2 r}, y_{2 r+1}\right) \rho\left(y_{2 r}, w_{r}\right)}{1+\rho\left(y_{2 r}, l\right)} \\
& +\psi_{5}\left(y_{0}\right) \frac{\rho\left(l, y_{2 r+1}\right) \rho\left(l, w_{r}\right)}{1+\rho\left(y_{2 r}, l\right)} \\
& +\psi_{6}\left(y_{0}\right) \frac{\rho\left(y_{2 r}, y_{2 r+1}\right) \rho\left(l, w_{r}\right)}{1+\rho\left(y_{2 r}, l\right)} \\
& +\psi_{7}\left(y_{0}\right) \frac{\rho\left(l, y_{2 r+1}\right) \rho\left(y_{2 r+1}, w_{r}\right)}{1+\rho\left(y_{2 r}, l\right)}
\end{aligned}
$$

which, on $r \rightarrow \infty$, reduced to

$$
\begin{gathered}
\rho\left(l, w_{r}\right) \preceq \psi_{3}\left(y_{0}\right) \rho\left(l, w_{r}\right) \\
\left|\rho\left(l, w_{r}\right)\right| \leq \psi_{3}\left(y_{0}\right)\left|\rho\left(l, w_{r}\right)\right| .
\end{gathered}
$$

Since $\psi_{3}\left(y_{0}\right)<1$, so $\left|\rho\left(l, w_{r}\right)\right| \rightarrow 0$ as $r \rightarrow \infty$. so we have $w_{r} \rightarrow l$ as $r \rightarrow \infty$. Since $\left[G_{2} l\right]_{\alpha}$ is closed, so $l \in\left[G_{2} l\right]_{\alpha}$. Similarly, it follows that $l \in\left[G_{1} l\right]_{\alpha}$. Thus we obtain that $G_{1}$ and $G_{2}$ have common fixed points.

Corollary 15. Let $(\mathscr{X}, \rho)$ be a complete complex-valued metric space and $G_{1}: \mathscr{X} \rightarrow \mathfrak{Q}(\mathscr{X})$ be fuzzy mapping with glb property. For each $y \in \mathscr{X}$ there exists $\alpha \in(0,1]$ such that $\left[G_{1} y\right]_{\alpha}$ is nonempty closed bounded subset of $\mathscr{X}$. Then there exists mappings $\psi_{i}: \mathscr{X} \rightarrow[0,1), i=1, \ldots, 7$ with

(i) $\psi_{i}(u) \preceq \psi_{i}(y), i=1,2, \ldots, 7$ for all $u \in\left[G_{1} y\right]_{\alpha}$ and $\forall y \in \mathscr{X}$ (ii) $\sum \psi_{i}(y)+2 \psi_{4}(y)<1$, where $i=1,2,3,6,7 \forall y \in \mathscr{X}$; and

$$
\begin{aligned}
& \Phi\left(\psi_{1}(y) \rho(y, w)+\psi_{2}(y) \rho\left(y,\left[G_{1} y\right]_{\alpha}\right)\right. \\
& +\psi_{3}(y) \rho\left(w,\left[G_{1} w\right]_{\alpha}\right) \\
& +\psi_{4}(y) \frac{\rho\left(y,\left[G_{1} y\right]_{\alpha}\right) \rho\left(y,\left[G_{1} w\right]_{\alpha}\right)}{1+\rho(y, w)} \\
& +\psi_{5}(y) \frac{\rho\left(w,\left[G_{1} y\right]_{\alpha}\right) \rho\left(w,\left[G_{1} w\right]_{\alpha}\right)}{1+\rho(y, w)} \\
& +\psi_{6}(y) \frac{\rho\left(y,\left[G_{1} y\right]_{\alpha}\right) \rho\left(w,\left[G_{1} w\right]_{\alpha}\right)}{1+\rho(y, w)} \\
& \left.+\psi_{7}(y) \frac{\rho\left(w,\left[G_{1} y\right]_{\alpha}\right) \rho\left(y,\left[G_{1} w\right]_{\alpha}\right)}{1+\rho(y, w)}\right) \\
& \quad \in s\left(\left[G_{1} y\right]_{\alpha},\left[G_{1} w\right]_{\alpha}\right),
\end{aligned}
$$

for some $\Phi \in \Psi$ and for all $y, w \in \mathscr{X}$. Then $G_{1}$ has a fuzzy fixed point.

Proof. Proof is immediate on setting $G_{1}=G_{2}$ in Theorem 14 .

Corollary 16. Let $(\mathscr{X}, \rho)$ be a complete complex-valued metric space and $G_{1}, G_{2}: \mathscr{X} \rightarrow \mathfrak{L}(\mathscr{X})$ be fuzzy mappings with glb property. For each $y \in \mathscr{X}$ there exists some $\alpha \in(0,1]$ such that $\left[G_{1} y\right]_{\alpha},\left[G_{2} y\right]_{\alpha}$, nonempty closed bounded subsets of $\mathscr{X}$. Then there exist mappings $\varrho, \sigma, \gamma, \xi, \zeta, \lambda, \eta: \mathscr{X} \rightarrow[0,1)$ with

(i) $\varrho(u) \preceq \varrho(y), \sigma(u) \preceq \sigma(y), \gamma(u) \preceq \gamma(y), \xi(u) \preceq \xi(y)$, $\zeta(u) \preceq \zeta(y), \lambda(u) \preceq \lambda(y), \eta(u) \preceq \eta(y)$ for all $u \in$ $\left[G_{1} y\right]_{\alpha}$ and $y \in \mathscr{X}$

(ii) $\varrho(v) \preceq \varrho(w), \sigma(v) \preceq \sigma(w), \gamma(v) \preceq \gamma(w), \xi(v) \preceq \xi(w)$, $\zeta(v) \preceq \zeta(w), \lambda(v) \preceq \lambda(w), \eta(v) \preceq \eta(w)$ for all $v \in$ $\left[G_{2} w\right]_{\alpha}$ and $w \in \mathscr{X}$;

(iii) $\varrho(y)+\sigma(y)+\gamma(y)+2 \xi(y)+\zeta(y)+\lambda(y)+\eta(y) \preceq 1 \forall y \epsilon$ $X$; and

$$
\begin{aligned}
\varrho(y) & \rho(y, w)+\sigma(y) \rho\left(y,\left[G_{1} y\right]_{\alpha}\right) \\
& +\gamma(y) \rho\left(w,\left[G_{2} w\right]_{\alpha}\right) \\
& +\xi(y) \frac{\rho\left(y,\left[G_{1} y\right]_{\alpha}\right) \rho\left(y,\left[G_{2} w\right]_{\alpha}\right)}{1+\rho(y, w)} \\
& +\zeta(y) \frac{\rho\left(w,\left[G_{1} y\right]_{\alpha}\right) \rho\left(w,\left[G_{2} w\right]_{\alpha}\right)}{1+\rho(y, w)} \\
& +\lambda(y) \frac{\rho\left(y,\left[G_{1} y\right]_{\alpha}\right) \rho\left(w,\left[G_{2} w\right]_{\alpha}\right)}{1+\rho(y, w)} \\
& +\eta(x) \frac{\rho\left(w,\left[G_{1} y\right]_{\alpha}\right) \rho\left(y,\left[G_{2} w\right]_{\alpha}\right)}{1+\rho(y, w)} \\
\epsilon & s\left(\left[G_{1} y\right]_{\alpha},\left[G_{2} w\right]_{\alpha}\right),
\end{aligned}
$$

$\forall y, w \in X$; then $G_{1}$ and $G_{2}$ have a common fuzzy fixed point. 
Proof. It can be easily proven by letting $\Phi(t)=p t$ where $p \in(0,1)$ in Theorem 14 with $\varrho(y)=p \psi_{1}(y), \sigma(y)=$ $p \psi_{2}(y), \gamma(y)=p \psi_{3}(y), \xi(y)=p \psi_{4}(y), \zeta(y)=p \psi_{5}(y)$, $\lambda(y)=p \psi_{6}(y), \eta(y)=p \psi_{7}(y)$.

Corollary 17. Suppose $(\mathscr{X}, \rho)$ is a complete complex-valued metric space and $G_{1}, G_{2}: \mathscr{X} \rightarrow \mathfrak{L}(\mathscr{X})$ are fuzzy mappings enjoying glb property. For each $y \in \mathscr{X}$ there exists some $\alpha \in(0,1]$ such that $\left[G_{1} y\right]_{\alpha},\left[G_{2} y\right]_{\alpha}$, nonempty closed bounded subsets of $\mathscr{X}$ with

$$
\begin{gathered}
\varrho \rho(y, w)+\sigma \rho\left(y,\left[G_{1} y\right]_{\alpha}\right)+\gamma \rho\left(w,\left[G_{2} w\right]_{\alpha}\right) \\
+\xi \frac{\rho\left(y,\left[G_{1} y\right]_{\alpha}\right) \rho\left(y,\left[G_{2} w\right]_{\alpha}\right)}{1+\rho(y, w)} \\
+\zeta \frac{\rho\left(w,\left[G_{1} y\right]_{\alpha}\right) \rho\left(w,\left[G_{2} w\right]_{\alpha}\right)}{1+\rho(y, w)} \\
+\lambda \frac{\rho\left(y,\left[G_{1} y\right]_{\alpha}\right) \rho\left(w,\left[G_{2} w\right]_{\alpha}\right)}{1+\rho(y, w)} \\
+\eta \frac{\rho\left(w,\left[G_{1} y\right]_{\alpha}\right) \rho\left(y,\left[G_{2} w\right]_{\alpha}\right)}{1+\rho(y, w)} \\
\epsilon s\left(\left[G_{1} y\right]_{\alpha},\left[G_{2} w\right]_{\alpha}\right),
\end{gathered}
$$

for all $y, w \in X$ and $\varrho, \sigma, \gamma, \xi, \zeta, \lambda, \eta$ are nonnegative reals with $\varrho+\sigma+\gamma+2 \xi+\zeta+\lambda+\eta<1$. Then $G_{1}$ and $G_{2}$ have a common fuzzy fixed point.

Proof. It can be easily proven by setting $\varrho(y)=\varrho, \sigma(y)=\sigma$, $\gamma(y)=\gamma, \xi(y)=\xi, \zeta(y)=\zeta, \lambda(y)=\lambda, \eta(y)=\eta$ in Corollary 16 with $\varrho, \sigma, \gamma, \xi, \zeta, \lambda, \eta$ being nonnegative reals such that $\varrho+\sigma+\gamma+2 \xi+\zeta+\lambda+\eta<1$.

Using Remark 11 we get the following corollaries from Theorem 14.

Corollary 18. Suppose $(X, \rho)$ is a complete metric space and $G_{1}, G_{2}: \mathscr{X} \rightarrow \mathfrak{L}(\mathscr{X})$ are fuzzy mappings with glb property. For each $y \in \mathscr{X}$ related to some $\alpha \in(0,1]$ there exists $\left[G_{1} y\right]_{\alpha},\left[G_{2} y\right]_{\alpha}$, nonempty closed bounded subsets of $X$. Then there exist mappings $\psi_{i}: \mathscr{X} \rightarrow[0,1), i=1,2, \ldots, 7$ such that

(i) $\psi_{i}(u) \leq \psi_{i}(y)$, for all $u \in\left[G_{1} y\right]_{\alpha}$ and $\forall y \in \mathscr{X}$;

(ii) $\psi_{i}(v) \leq \psi_{i}(y)$, for all $v \in\left[G_{2} y\right]_{\alpha}$ and $\forall y \in \mathscr{X}$;

(iii) $\sum \psi_{i}(y)+2 \psi_{4}(y)<1, i=1,2,3,5,6,7 \forall y \in \mathscr{X}$; and

$$
\begin{aligned}
& H\left(\left[G_{1} y\right]_{\alpha},\left[G_{2} w\right]_{\alpha}\right) \leq \Phi\left(\psi_{1}(y) \rho(y, w)\right. \\
& +\psi_{2}(y) \rho\left(y,\left[G_{1} y\right]_{\alpha}\right)+\psi_{3}(y) \rho\left(w,\left[G_{2} w\right]_{\alpha}\right) \\
& \quad+\psi_{4}(y) \frac{\rho\left(y,\left[G_{1} y\right]_{\alpha}\right) \rho\left(y,\left[G_{2} w\right]_{\alpha}\right)}{1+\rho(y, w)} \\
& \quad+\psi_{5}(y) \frac{\rho\left(w,\left[G_{1} y\right]_{\alpha}\right) \rho\left(w,\left[G_{2} w\right]_{\alpha}\right)}{1+\rho(y, w)}
\end{aligned}
$$

$$
\begin{aligned}
& +\psi_{6}(y) \frac{\rho\left(y,\left[G_{1} y\right]_{\alpha}\right) \rho\left(w,\left[G_{2} w\right]_{\alpha}\right)}{1+\rho(y, w)} \\
& \left.+\psi_{7}(y) \frac{\rho\left(w,\left[G_{1} y\right]_{\alpha}\right) \rho\left(y,\left[G_{2} w\right]_{\alpha}\right)}{1+\rho(y, w)}\right),
\end{aligned}
$$

for some $\Phi \in \Psi$ and for all $y, w \in \mathscr{X}$. Then $G_{1}$ and $G_{2}$ have a common fuzzy fixed point.

Corollary 19. Suppose $(\mathscr{X}, \rho)$ is complete metric space and $G_{1}, G_{2}: \mathscr{X} \rightarrow \mathfrak{Q}(\mathscr{X})$ is fuzzy mappings with glb property. For each $y \in \mathscr{X}$ there exists some $\alpha \in(0,1]$ such that $\left[G_{1} y\right]_{\alpha},\left[G_{2} y\right]_{\alpha}$, nonempty closed bounded subsets of $\mathscr{X}$ such that

$$
\begin{aligned}
H\left(\left[G_{1} y\right]_{\alpha},\left[G_{2} w\right]_{\alpha}\right) & \\
\leq & \varrho \rho(y, w)+\sigma \rho\left(y,\left[G_{1} y\right]_{\alpha}\right)+\gamma \rho\left(w,\left[G_{2} w\right]_{\alpha}\right) \\
& +\xi \frac{\rho\left(y,\left[G_{1} y\right]_{\alpha}\right) \rho\left(y,\left[G_{2} w\right]_{\alpha}\right)}{1+\rho(y, w)} \\
& +\zeta \frac{\rho\left(w,\left[G_{1} y\right]_{\alpha}\right) \rho\left(w,\left[G_{2} w\right]_{\alpha}\right)}{1+\rho(y, w)} \\
& +\lambda \frac{\rho\left(y,\left[G_{1} y\right]_{\alpha}\right) \rho\left(w,\left[G_{2} w\right]_{\alpha}\right)}{1+\rho(y, w)} \\
& +\eta \frac{\rho\left(w,\left[G_{1} y\right]_{\alpha}\right) \rho\left(y,\left[G_{2} w\right]_{\alpha}\right)}{1+\rho(y, w)}
\end{aligned}
$$

for all $y, w \in \mathscr{X}$ and $\varrho, \sigma, \gamma, \xi, \zeta, \lambda, \eta$ are nonnegative reals with $\varrho+\sigma+\gamma+2 \xi+\zeta+\lambda+\eta<1$. Then $G_{1}$ and $G_{2}$ have a common fuzzy fixed point.

Proof. By putting $\varrho(y)=\psi_{1}(y), \sigma(y)=\psi_{2}(y), \gamma(y)=$ $\psi_{3}(y), \xi(y)=\psi_{4}(y), \zeta(y)=\psi_{5}(y), \lambda(y)=\psi_{6}(y), \eta(y)=$ $\psi_{7}(y)$ in Corollary 18 , it can be easily proven.

\section{Application}

Theorem 20. Let $(\mathscr{X}, \rho)$ be a complete complex-valued metric space and $G_{1}, G_{2}: \mathscr{X} \rightarrow \mathscr{C} \mathscr{B}(\mathscr{X})$ be multivalued mapping with glb property. If there exist mappings $\psi_{i}: \mathscr{X} \rightarrow[0,1), i=$ $1,2, \ldots, 7$ such that

(i) $\psi_{i}(u) \preceq \psi_{i}(y)$ for all $u \in G_{1} y$ and $\forall y \in \mathscr{X}$;

(ii) $\psi_{i}(v) \preceq \psi_{i}(y)$ for all $v \in G_{2} y$ and $\forall y \in \mathscr{X}$;

(iii) $\psi_{i}(y)+2 \psi_{4}(y)<1, i=1,2,3,5,6,7 \forall y \in \mathscr{X}$; and

$$
\begin{aligned}
& \Phi\left(\psi_{1}(y) \rho(y, w)+\psi_{2}(y) \rho\left(y, G_{1} y\right)\right. \\
& \quad+\psi_{3}(y) \rho\left(w, G_{2} w\right) \\
& \quad+\psi_{4}(y) \frac{\rho\left(y, G_{1} y\right) \rho\left(y, G_{2} w\right)}{1+\rho(y, w)}
\end{aligned}
$$




$$
\begin{aligned}
& +\psi_{5}(y) \frac{\rho\left(w, G_{1} y\right) \rho\left(w, G_{2} w\right)}{1+\rho(y, w)} \\
& +\psi_{6}(y) \frac{\rho\left(y, G_{1} y\right) \rho\left(w, G_{2} w\right)}{1+\rho(y, w)} \\
& \left.+\psi_{7}(y) \frac{\rho\left(w, G_{1} y\right) \rho\left(y, G_{2} w\right)}{1+\rho(y, w)}\right) \in s\left(G_{1} y, G_{2} w\right),
\end{aligned}
$$

for some $\Phi \in \Psi$ and for all $y, w \in \mathscr{X}$, then $G_{1}$ and $G_{2}$ have a common fixed point.

Proof. Let the fuzzy mapping $S, T: \mathscr{X} \rightarrow \mathfrak{Q}(\mathscr{X})$ be defined by

$$
\begin{gathered}
S y= \begin{cases}\alpha & \text { if } y \in G_{1} y \\
0 & \text { if } y \notin G_{1} y .\end{cases} \\
T y= \begin{cases}\alpha & \text { if } y \in G_{2} y \\
0 & \text { if } y \notin G_{2} y .\end{cases}
\end{gathered}
$$

Then for any $\alpha \in(0,1, S y]_{\alpha}=G_{1} y$ and $[T y]_{\alpha}=G_{2} y$.

Since for every $y, w \in \mathscr{X}, s\left([S y]_{\alpha},[T w]_{\alpha}\right)=s\left(G_{1} y, G_{2} w\right)$, therefore Theorem 14 can be applied to obtain some points $u \in \mathscr{X}$ such that $u \in G_{1}(u) \cap G_{2}(u)$.

Corollary 21. Let $(\mathscr{X}, \rho)$ be a complete complex-valued metric space and $G_{1}, G_{2}: \mathscr{X} \rightarrow \mathscr{C} \mathscr{B}(\mathscr{X})$ be multivalued mapping with glb property. Suppose there exist mappings $\psi_{i}: \mathscr{X} \rightarrow$ $[0,1), i=1,2, \ldots, 7$ such that

(i) $\psi_{i}(u) \preceq \psi_{i}(y)$ for all $u \in G_{1} y$ and $\forall y \in \mathscr{X}$;

(ii) $\psi_{i}(v) \preceq \psi_{i}(y)$ for all $v \in G_{2} y$ and $\forall y \in \mathscr{X}$;

(iii) $\psi_{i}(y)+2 \psi_{4}(y)<1, i=1,2,3,5,6,7 \forall y \in \mathscr{X}$; and

$$
\begin{aligned}
\psi_{1}(y) & \rho(y, w)+\psi_{2}(y) \rho\left(y, G_{1} y\right) \\
& +\psi_{3}(y) \rho\left(w, G_{2} w\right) \\
& +\psi_{4}(y) \frac{\rho\left(y, G_{1} y\right) \rho\left(y, G_{2} w\right)}{1+\rho(y, w)} \\
& +\psi_{5}(y) \frac{\rho\left(w, G_{1} y\right) \rho\left(w, G_{2} w\right)}{1+\rho(y, w)} \\
& +\psi_{6}(y) \frac{\rho\left(y, G_{1} y\right) \rho\left(w, G_{2} w\right)}{1+\rho(y, w)} \\
+ & \psi_{7}(y) \frac{\rho\left(w, G_{1} y\right) \rho\left(y, G_{2} w\right)}{1+\rho(y, w)} \\
\epsilon & s\left(G_{1} y, G_{2} w\right) .
\end{aligned}
$$

\section{Then $G_{1}$ and $G_{2}$ have a common fixed point.}

Proof. It can be proven by the same way as Corollary 16.
Remark 22. (i) Theorem 20 is actually Theorem 2.3 of [34].

(ii) By setting $\psi_{2}(y)=\psi_{3}(y)=\psi_{4}(y)=\psi_{5}(y)=$ $0, \psi_{1}(y)=\varrho, \psi_{6}(y)=\lambda, \psi_{7}(y)=\eta$ in Corollary 21, we get Theorem 9 of [13].

(iii) By setting $\psi_{1}(y)=\psi_{4}(y)=\psi_{5}(y)=\psi_{7}=0, \psi_{2}(y)=$ $\sigma, \psi_{3}(y)=\gamma, \psi_{6}(y)=\lambda$ in Corollary 21 we obtain Theorem 15 of [13].

(iv) By setting $\psi_{2}(y)=\psi_{3}(y)=0, \psi_{1}(y)=\varrho, \psi_{4}(y)=$ $\xi, \psi_{5}(y)=\zeta, \psi_{6}(y)=\lambda, \psi_{7}(y)=\eta$ in Corollary 21 we get Theorem 9 of [36].

(v) By setting $\sigma=\gamma=\xi=\zeta=0$ in Corollary 17, we get Theorem 12 of [25].

(vi) By setting $\varrho=\xi=\zeta=\eta=0$ in Corollary 17, we get Theorem 19 of [25].

Example 23. Let $\mathscr{X}=[0,1]$ and $\rho: \mathscr{X} \times \mathscr{X} \rightarrow \mathscr{C}$ be complexvalued metric space defined by $\rho(y, w)=|y-w| e^{i(\pi / 12)}$, for all $y, w \in \mathscr{X}$.

Let $\alpha \in(0,1]$ and $G_{1}, G_{2}: \mathscr{X} \rightarrow \mathfrak{Q}(\mathscr{X})$ be fuzzy mappings defined by

$$
\begin{aligned}
& G_{1}(0)(r)= \begin{cases}1 & \text { if } r=0 \\
\frac{1}{2} & \text { if } 0<r \leq \frac{y}{50} \\
0 & \text { if } \frac{y}{50}<r \leq 1,\end{cases} \\
& G_{2}(0)(r)= \begin{cases}1 & \text { if } r=0 \\
\frac{1}{6} & \text { if } 0<r \leq \frac{y}{150} \\
0 & \text { if } \frac{y}{150}<r \leq 1,\end{cases}
\end{aligned}
$$

if $y \neq 0$,

$$
\begin{aligned}
& G_{1}(y)(r)= \begin{cases}\alpha & \text { if } 0 \leq r \leq \frac{y}{75} \\
\frac{\alpha}{3} & \text { if } \frac{y}{75}<r \leq \frac{y}{10} \\
\frac{\alpha}{4} & \text { if } \frac{y}{10}<r \leq 1,\end{cases} \\
& G_{2}(y)(r)= \begin{cases}\alpha & \text { if } 0 \leq r \leq \frac{y}{40} \\
\frac{\alpha}{2} & \text { if } \frac{y}{40}<r \leq \frac{y}{20} \\
\frac{\alpha}{5} & \text { if } \frac{y}{20}<r \leq 1 .\end{cases}
\end{aligned}
$$

Let $\psi_{1}, \psi_{2}, \psi_{3}, \psi_{4}, \psi_{5}, \psi_{6}, \psi_{7}: \mathscr{X} \rightarrow[0,1)$ be defined by $\psi_{1}(y)=(y+1) / 74, \psi_{2}(y)=y / 10, \psi_{3}(y)=y / 20, \psi_{4}(y)=$ $y / 30, \psi_{5}(y)=y / 60, \psi_{6}(y)=y / 40$.

Then for $y=0,\left[G_{1} 0\right]_{1}=\left[G_{2} 0\right]_{1}=\{0\}$ and $\forall y, w \neq$ $0\left[G_{1} y\right]_{\alpha}=[0, y / 75]$ and $\left[G_{2} y\right]_{\alpha}=[0, y / 40]$.

And then

$$
\begin{aligned}
& \mathscr{W}_{w}\left(\left[G_{1} y\right]_{\alpha}\right)=\left\{\rho(w, u): u \in\left[0, \frac{y}{75}\right]\right\} \\
& \mathscr{W}_{w}\left(\left[G_{2} y\right]_{\alpha}\right)=\left\{\rho(w, u): u \in\left[0, \frac{y}{40}\right]\right\} .
\end{aligned}
$$


Let $\rho\left(w,\left[G_{1} w\right]_{\alpha}\right)$ and $\rho\left(w,\left[G_{2} w\right]_{\alpha}\right)$ be the greatest lower bound of $\mathscr{W}_{w}\left(\left[G_{1} y\right]_{\alpha}\right)$ and $\mathscr{W}_{w}\left(\left[G_{2} y\right]_{\alpha}\right)$. Then

$$
\begin{aligned}
& \rho\left(w,\left[G_{1} y\right]_{\alpha}\right)= \begin{cases}0 & \text { if } w \leq \frac{y}{75} \\
\left(w-\frac{y}{75}\right) e^{i(\pi / 12)} & \text { if } w>\frac{y}{75}\end{cases} \\
& \rho\left(y,\left[G_{2} w\right]_{\alpha}\right)= \begin{cases}0 & \text { if } y \leq \frac{y}{40} \\
\left(y-\frac{w}{40}\right) e^{i(\pi / 12)} & \text { if } x>\frac{w}{40} ;\end{cases}
\end{aligned}
$$

also $\rho\left(y,\left[G_{1} y\right]_{\alpha}\right)=(74 y / 75) e^{i(\pi / 12)}$, and $\rho\left(w,\left[G_{2} w\right]_{\alpha}\right)=$ $(39 w / 40) e^{i(\pi / 12)}$.

It can be easily verified that $\psi_{i}(u) \leq \psi_{i}(w), \forall u \in\left[G_{1} y\right]_{\alpha}$ and $\psi_{i}(v) \leq \psi_{i}(w), \forall v \in\left[G_{2} y\right]_{\alpha}$. Moreover if $\varrho_{y w} \in \mathfrak{C}$ such that

$$
\varpi_{y w}=\left|\frac{y}{75}-\frac{w}{40}\right| \sqrt{2} e^{i(\pi / 12)},
$$

then

$$
s\left(\left[G_{1} y\right]_{\alpha},\left[G_{2} w\right]_{\alpha}\right)=\left\{\omega \in \mathbb{C}: \Phi_{y w} \preceq \omega\right\} .
$$

Consider

$$
\begin{aligned}
& \Phi\left(\psi_{1}(y)|\rho(y, w)|+\psi_{2}(y)\left|\rho\left(y,\left[G_{1} y\right]_{\alpha}\right)\right|\right. \\
& \quad+\psi_{3}(y)\left|\rho\left(w,\left[G_{2} w\right]_{\alpha}\right)\right| \\
& \quad+\psi_{4}(y) \frac{\left|\rho\left(y,\left[G_{1} y\right]_{\alpha}\right)\right|\left|\rho\left(y,\left[G_{2} w\right]_{\alpha}\right)\right|}{1+|\rho(y, w)|} \\
& \quad+\psi_{5}(y) \frac{\left|\rho\left(w,\left[G_{1} y\right]_{\alpha}\right)\right|\left|\rho\left(w,\left[G_{2} w\right]_{\alpha}\right)\right|}{1+|\rho(y, w)|} \\
& \quad+\psi_{6}(y) \frac{\left|\rho\left(y,\left[G_{1} y\right]_{\alpha}\right)\right|\left|\rho\left(w,\left[G_{2} w\right]_{\alpha}\right)\right|}{1+|\rho(y, w)|} \\
& \left.\quad+\psi_{7}(y) \frac{\left|\rho\left(w,\left[G_{1} y\right]_{\alpha}\right)\right|\left|\rho\left(y,\left[G_{2} w\right]_{\alpha}\right)\right|}{1+|\rho(y, w)|}\right)
\end{aligned}
$$

then clearly for $\psi_{1}(y)=(y+1) / 74, \psi_{2}(y)=y / 10, \psi_{3}(y)=$ $y / 20, \psi_{4}(y)=y / 30, \psi_{5}(y)=y / 60, \psi_{6}(y)=y / 40, \psi_{7}=$ $y / 25$ and $\Phi(t)=74 t / 75$.

$$
\begin{aligned}
& \left|\frac{y}{75}-\frac{w}{40}\right| \leq \frac{74}{75}\left(\frac{y+1}{74}|y-w|+\frac{y}{10}\left|\frac{74 y}{75}\right|\right. \\
& +\frac{y}{20}\left|\frac{39 w}{40}\right|+\frac{y}{30} \frac{|74 y / 75||y-w / 40|}{1+|y-w|} \\
& +\frac{y}{60} \frac{|w-y / 75||39 w / 40|}{1+|y-w|} \\
& +\frac{y}{40} \frac{|74 y / 75||39 w / 40|}{1+|y-w|} \\
& \left.+\frac{y}{75} \frac{|w-y / 75||y-w / 40|}{1+|y-w|}\right)
\end{aligned}
$$

which can be easily calculated by

$$
\begin{aligned}
\frac{74}{75}\left(\frac{y+1}{74}|y-w|\right) & =\frac{74}{25}\left(\frac{1}{74}\left|y^{2}+y-w-y w\right|\right) \\
& \geq \frac{1}{75}(|y-w-y w|) \\
& =\left|\frac{y}{75}-\frac{w(1+y)}{75}\right| \geq\left|\frac{y}{75}-\frac{w}{75}\right| \\
& \geq\left|\frac{y}{75}-\frac{w}{40}\right| .
\end{aligned}
$$

The remaining terms of (59) are nonzero reals. Consequently we can obtain

$$
\begin{aligned}
& \Phi\left(\psi_{1}(y) \rho(y, w)+\psi_{2}(y) \rho\left(y,\left[G_{1} y\right]_{\alpha}\right)\right. \\
& +\psi_{3}(y) \rho\left(w,\left[G_{2} w\right]_{\alpha}\right) \\
& +\psi_{4}(y) \frac{\rho\left(y,\left[G_{1} y\right]_{\alpha}\right) \rho\left(y,\left[G_{2} w\right]_{\alpha}\right)}{1+\rho(y, w)} \\
& +\psi_{5}(y) \frac{\rho\left(w,\left[G_{1} y\right]_{\alpha}\right) \rho\left(w,\left[G_{2} w\right]_{\alpha}\right)}{1+\rho(y, w)} \\
& +\psi_{6}(y) \frac{\rho\left(y,\left[G_{1} y\right]_{\alpha}\right) \rho\left(w,\left[G_{2} w\right]_{\alpha}\right)}{1+\rho(y, w)} \\
& \left.+\psi_{7}(y) \frac{\rho\left(w,\left[G_{1} y\right]_{\alpha}\right) \rho\left(y,\left[G_{2} w\right]_{\alpha}\right)}{1+\rho(y, w)}\right) \geq \Phi_{y w} .
\end{aligned}
$$

Therefore

$$
\begin{aligned}
& \Phi\left(\psi_{1}(y) \rho(y, w)+\psi_{2}(y) \rho\left(y,\left[G_{1} y\right]_{\alpha}\right)\right. \\
& +\psi_{3}(y) \rho\left(w,\left[G_{2} w\right]_{\alpha}\right) \\
& +\psi_{4}(y) \frac{\rho\left(y,\left[G_{1} y\right]_{\alpha}\right) \rho\left(y,\left[G_{2} w\right]_{\alpha}\right)}{1+\rho(y, w)} \\
& +\psi_{5}(y) \frac{\rho\left(w,\left[G_{1} y\right]_{\alpha}\right) \rho\left(w,\left[G_{2} w\right]_{\alpha}\right)}{1+\rho(y, w)} \\
& +\psi_{6}(y) \frac{\rho\left(y,\left[G_{1} y\right]_{\alpha}\right) \rho\left(w,\left[G_{2} w\right]_{\alpha}\right)}{1+\rho(y, w)} \\
& \left.\quad+\psi_{7}(y) \frac{\rho\left(w,\left[G_{1} y\right]_{\alpha}\right) \rho\left(y,\left[G_{2} w\right]_{\alpha}\right)}{1+\rho(y, w)}\right) \\
& \quad \in s\left(\left[G_{1} y\right]_{\alpha},\left[G_{2} w\right]_{\alpha}\right) .
\end{aligned}
$$

Hence all conditions of Theorem 14 are satisfied by $G_{1}, G_{2}$; therefore there exists $0 \in \mathscr{X}$ such that $0 \in\left[G_{1} 0\right]_{\alpha} \cap\left[G_{2} 0\right]_{\alpha}$.

\section{Conflicts of Interest}

The authors declare that they have no conflicts of interest. 


\section{References}

[1] S. Banach, "Sur les opérations dans les ensembles abstraits et leur application aux équations intégrales," Fundamenta Mathematicae, vol. 3, pp. 133-181, 1922.

[2] A. Azam, N. Mehmood, M. Rashid, and S. Radenović, "Fuzzy fixed point theorems in ordered cone metric spaces," Filomat, vol. 29, no. 4, pp. 887-896, 2015.

[3] A. Azam, M. Arshad, and I. Beg, "Banach contraction principle on cone rectangular metric spaces," Applicable Analysis and Discrete Mathematics, vol. 3, no. 2, pp. 236-241, 2009.

[4] L. B. Ćirić, "A generalization of Banach's contraction principle," Proceedings of the American Mathematical Society, vol. 45, pp. 267-273, 1974.

[5] T. Dov senovi'c, D. s. Raki'c, B. Cari'c, and S. Radenovi'c, "Multivalued generalizations of fixed point results in fuzzy metric spaces," Lithuanian Association of Nonlinear Analysts. Nonlinear Analysis: Modelling and Control, vol. 21, no. 2, pp. 211222, 2016.

[6] E. Karapinar and R. P. Agarwal, "Further fixed point results on G metric space," Fixed Point Theory Appl, pp. 107-173, 2015.

[7] S. Radenovic, "Common fixed points under contractive conditions in cone metric spaces," Computers \& Mathematics with Applications, vol. 58, no. 6, pp. 1273-1278, 2009.

[8] S. Radenovi'c and B. E. Rhoades, "Fixed point theorem for two non-self mappings in cone metric spaces," Computers \& Mathematics with Applications, vol. 57, no. 10, pp. 1701-1707, 2009.

[9] B. K. Dass and S. Gupta, "An extension of Banach contraction principle through rational expression," Indian Journal of Pure and Applied Mathematics, vol. 6, no. 12, pp. 1455-1458, 1975.

[10] A. Azam, B. Fisher, and M. Khan, "Common fixed point theorems in complex valued metric spaces," Numerical Functional Analysis and Optimization, vol. 32, no. 3, pp. 243-253, 2011.

[11] K. Sitthikul and S. Saejung, "Some fixed point theorems in complex valued metric spaces," Fixed Point Theory and Applications, vol. 2012, article no. 189, 2012.

[12] F. Rouzkard and M. Imdad, "Some common fixed point theorems on complex valued metric spaces," Computers \& Mathematics with Applications, vol. 64, no. 6, pp. 1866-1874, 2012.

[13] J. Ahmad, C. Klin-Eam, and A. Azam, "Common fixed points for multivalued mappings in complex valued metric spaces with applications," Abstract and Applied Analysis, vol. 2013, Article ID 854965, 12 pages, 2013.

[14] A. Azam, J. Ahmad, and P. Kumam, "Common fixed point theorems for multi-valued mappings in complex-valued metric spaces," Journal of Inequalities and Applications, vol. 2013, article 578, p. 12, 2013.

[15] W. Sintunavarat and P. Kumam, "Generalized common fixed point theorems in complex valued metric spaces and applications," Journal of Inequalities and Applications, vol. 2012, article no. 84, 2012.

[16] S. a. Heilpern, "Fuzzy mappings and fixed point theorem," Journal of Mathematical Analysis and Applications, vol. 83, no. 2, pp. 566-569, 1981.

[17] J. Nadler, "Multi-valued contraction mappings," Pacific Journal of Mathematics, vol. 30, pp. 475-488, 1969.

[18] A. Azam, "Fuzzy fixed points of fuzzy mappings via a rational inequality," Hacettepe Journal of Mathematics and Statistics, vol. 40, no. 3, pp. 421-431, 2011.
[19] S. C. Arora and V. Sharma, "Fixed point theorems for fuzzy mappings," Fuzzy Sets and Systems, vol. 110, no. 1, pp. 127-130, 2000.

[20] A. Azam and I. Beg, "Common fuzzy fixed points for fuzzy mappings," Fixed Point Theory and Applications, vol. 2013, article no. 14, pp. 1-11, 2013.

[21] R. K. Bose and D. Sahani, "Fuzzy mappings and fixed point theorems," Fuzzy Sets and Systems, vol. 21, no. 1, pp. 53-58, 1987.

[22] V. D. Estruch and A. Vidal, "A note on fixed fuzzy points for fuzzy mappings," vol. 32, no. suppl. 2, pp. 39-45 (2002).

[23] V. n. Gregori and J. Pastor, "A fixed point theorem for fuzzy contraction mappings," vol. 30, no. suppl., pp. 103-109.

[24] D. Turkoglu and B. E. Rhoades, "A fixed fuzzy point for fuzzy mapping in complete metric spaces," Mathematical Communications, vol. 10, no. 2, pp. 115-121, 2005.

[25] M. A. Kutbi, J. Ahmad, A. Azam, and N. Hussain, "On fuzzy fixed points for fuzzy maps with generalized weak property," Journal of Applied Mathematics, vol. 2014, Article ID 549504, 12 pages, 2014.

[26] O. Kaleva, "Fuzzy differential equations," Fuzzy Sets and Systems, vol. 24, no. 3, pp. 301-317, 1987.

[27] J. J. Nieto, "The cauchy problem for continuous fuzzy differential equations," Fuzzy Sets and Systems, vol. 102, no. 2, pp. 259-262, 1999.

[28] S. Song, L. Guo, and C. Feng, "Global existence of solutions to fuzzy differential equations," Fuzzy Sets and Systems, vol. 115, no. 3, pp. 371-376, 2000.

[29] S. Seikkala, "On the fuzzy initial value problem," Fuzzy Sets and Systems, vol. 24, no. 3, pp. 319-330, 1987.

[30] P. Balasubramaniam and S. Muralisankar, "Existence and uniqueness of fuzzy solution for the nonlinear fuzzy integrodifferential equations," Applied Mathematics Letters, vol. 14, no. 4, pp. 455$462,2001$.

[31] H. V. Long, N. T. Son, and N. V. Hoa, "Fuzzy fractional partial differential equations in partially ordered metric spaces," Iranian Journal of Fuzzy Systems, vol. 14, no. 2, pp. 107-126, 2017.

[32] H. V. Long, N. T. K. Son, and R. Rodríguez-López, "Some generalizations of fixed point theorems in partially ordered metric spaces and applications to partial differential equations with uncertainty," Vietnam Journal of Mathematics, pp. 1-25, 2017.

[33] H. V. Long, J. J. Nieto, and N. T. K. Son, "New approach for studying nonlocal problems related to differential systems and partial differential equations in generalized fuzzy metric spaces," Fuzzy Sets and Systems, vol. 331, no. 15, pp. 26-46, 2016.

[34] V. Joshi, N. Singh, and D. Singh, " $\Phi$-contractive multivalued mappings in complex valued metric spaces and remarks on some recent papers," Cogent Mathematics, 2016.

[35] W. Shatanawi, V. Ć. Rajić, S. Radenović, and A. Al-Rawashdeh, "Mizoguchi-Takahashi-type theorems in tvs-cone metric spaces," Fixed Point Theory and Applications, vol. 2012, article no. 106, pp. 1-7, 2012.

[36] M. A. Kutbi, J. Ahmad, A. Azam, and A. S. Al-Rawashdeh, "Generalized common fixed point results via greatest lower bound property," Journal of Applied Mathematics, vol. 2014, Article ID 265865, 2014. 


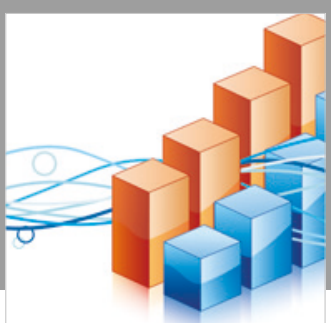

Advances in

Operations Research

\section{-n-m}
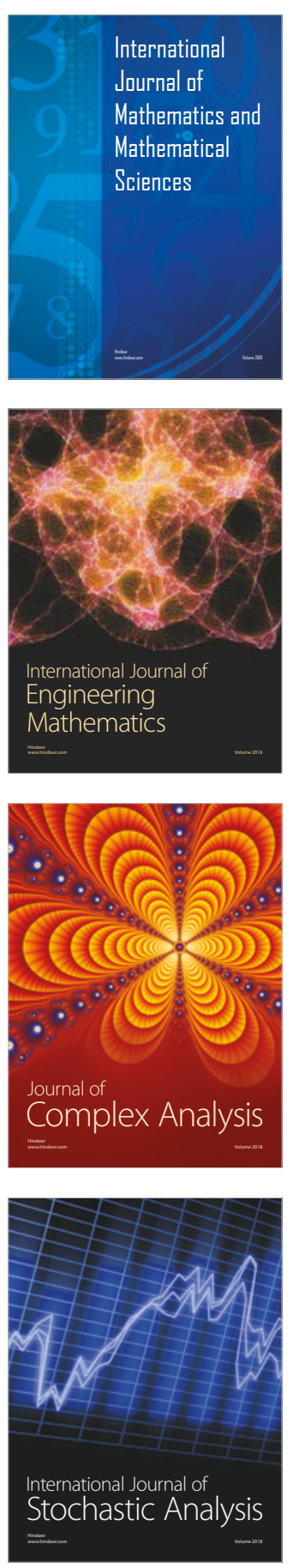
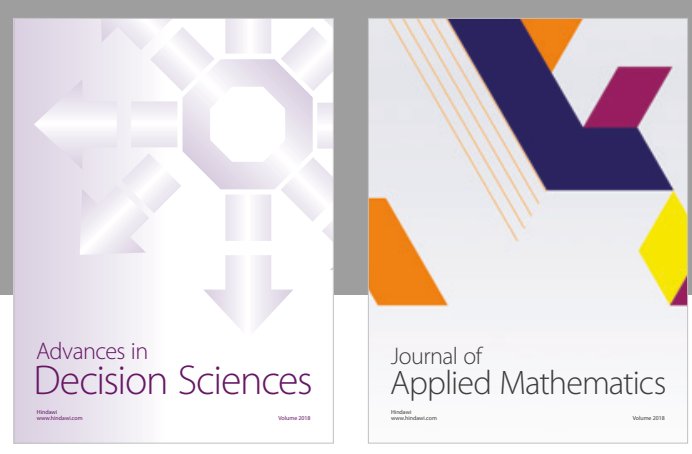

Journal of

Applied Mathematics
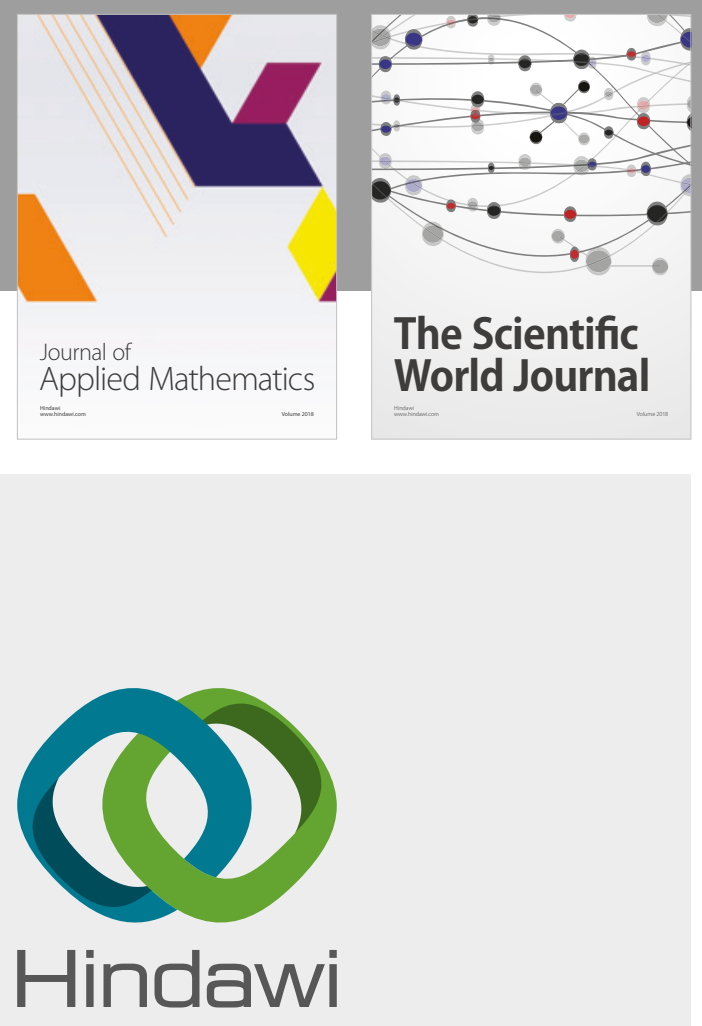

Submit your manuscripts at

www.hindawi.com

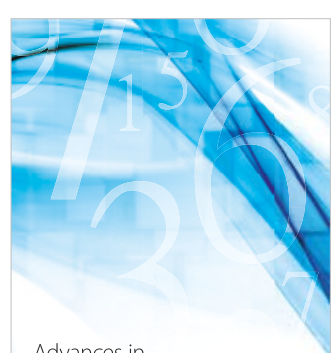

Advances in
Numerical Analysis
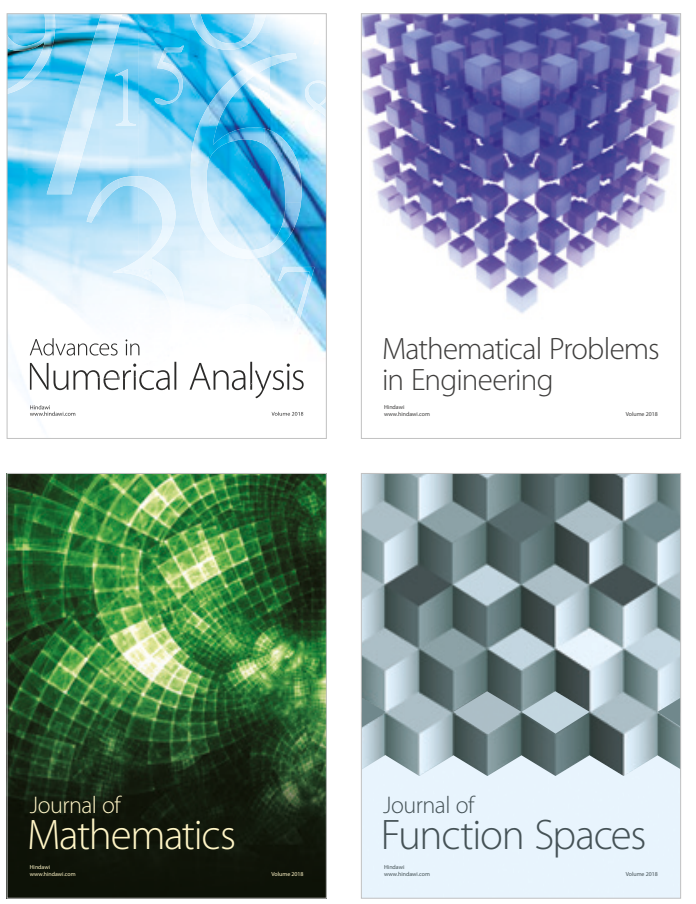

Mathematical Problems in Engineering

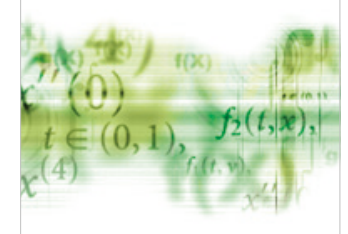

International Journal of

Differential Equations

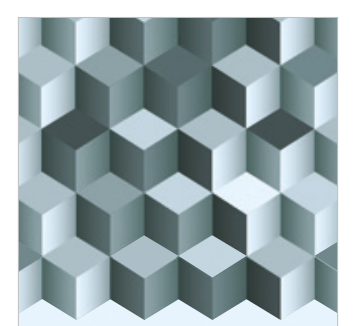

Journal of

Function Spaces
The Scientific

World Journal

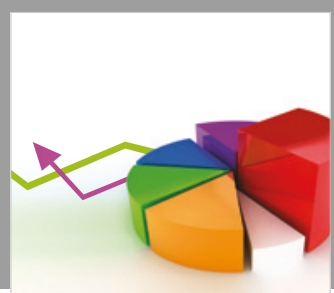

Journal of

Probability and Statistics
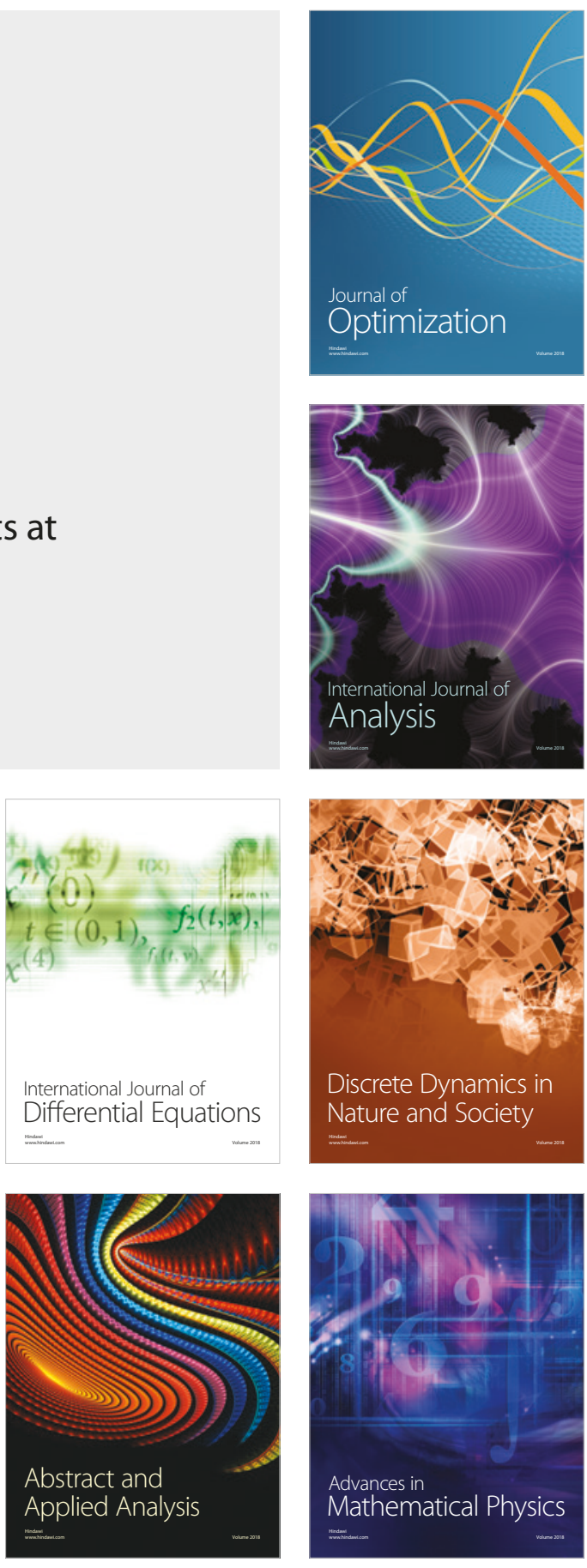